\title{
ON THE AVERAGE $L^{q}$-DIMENSIONS OF TYPICAL MEASURES BELONGING TO THE GROMOV-HAUSDORFF-PROHOROFF SPACE. THE LIMITING CASES: $q=1$ AND $q=\infty$
}

\author{
Lars Olsen \\ University of St. Andrews, Department of Mathematics \\ St. Andrews, Fife KY16 9SS, Scotland; lo@st-and.ac.uk
}

\begin{abstract}
We study the average $L^{q}$-dimensions of typical Borel probability measures belonging to the Gromov-Hausdorff-Prohoroff space (of all Borel probability measures with compact supports) equipped with the Gromov-Hausdorff-Prohoroff metric. Previously the lower and upper average $L^{q}$-dimensions of a typical measure $\mu$ have been found for $q \in(1, \infty)$. In this paper we determine the lower and upper average $L^{q}$-dimensions of a typical measure $\mu$ in the two limiting cases: $q=1$ and $q=\infty$. In particular, we prove that a typical measure $\mu$ is as irregular as possible: for $q=1$ and $q=\infty$, the lower average $L^{q}$-dimension attains the smallest possible value, namely 0 , and the upper average $L^{q}$-dimension attains the largest possible value, namely $\infty$. The proofs rely on some non-trivial semi-continuity properties of $L^{q}$-dimensions that may be of interest in their own right.
\end{abstract}

\section{Statement of results}

Recall that a subset $E$ of a (complete) metric space $M$ is called co-meagre if its complement is meagre, and we say that a typical element $x \in M$ has property $\mathrm{P}$ if the set $E=\{x \in M \mid x$ has property $\mathrm{P}\}$ is co-meagre, see Oxtoby [Oxt] for more details. For $1<q<\infty$, the $L^{q}$-dimensions, also sometimes called the Renyi entropies, of a typical Borel probability measures belonging to the Gromov-HausdorffProhoroff space (of all Borel probability measures with compact support) equipped with the Gromov-Hausdorff-Prohoroff metric have recently been computed. In the present paper we complete this study by computing the $L^{q}$-dimensions/Renyi entropies of a typical Borel probability measures belonging to the Gromov-HausdorffProhoroff space equipped with the Gromov-Hausdorff-Prohoroff metric for the two limiting cases, namely, $q=1$ and $q=\infty$. In Section 1.1 we recall the definition of the Gromov-Hausdorff-Prohoroff space and the Gromov-Hausdorff-Prohoroff metric, and in Section 1.2 we recall the definitions of the $L^{q}$-dimensions. The main results are presented in Section 1.3 and Section 1.4. We note that the proofs of our main results rely on some non-trivial semi-continuity properties of $L^{q}$-dimensions/Renyi entropies that may be of interest in their own right; these semi-continuity results are stated and discussed in Section 1.5.

1.1. The Gromov-Hausdorff-Prohoroff space $\boldsymbol{P}_{\mathrm{GHP}}$ and the GromovHausdorff-Prohoroff metric $\boldsymbol{d}_{\mathrm{GHP}}$. For a compact metric space $X$, we denote

https://doi.org/10.5186/aasfm.2020.4535

2010 Mathematics Subject Classification: Primary 28A78, 28A80.

Key words: $L^{q}$-dimension, Gromov-Hausdorff-Prohoroff space, Gromov-Hausdorff-Prohoroff metric, Hölder mean, Cesàro mean, Baire category. 
the family of all Borel probability measures on $X$ by $\mathcal{P}(X)$, i.e. we write

$$
\mathcal{P}(X)=\{\mu \mid \mu \text { is a Borel probability measure on } X\} .
$$

The pre-Gromov-Hausdorff-Prohoroff space $\mathcal{P}_{\mathrm{GHP}}$ is now defined by

$$
\begin{aligned}
\mathcal{P}_{\mathrm{GHP}} & =\bigcup_{\substack{X \text { is a compact } \\
\text { metric space }}} \mathcal{P}(X) \\
& =\{\mu \mid \mu \text { is a Borel probability measure on a compact metric space }\} .
\end{aligned}
$$

Next, we define the equivalence relation $\sim$ in $\mathcal{P}_{\mathrm{GHP}}$ as follows. Namely, for $\mu, \nu \in \mathcal{P}_{\mathrm{GHP}}$, we write

$\mu \sim \nu \Longleftrightarrow$ there is a bijective isometry $f: \operatorname{supp} \mu \rightarrow \operatorname{supp} \nu$ such that $\nu=\mu \circ f^{-1}$.

It is clear that $\sim$ is an equivalence relation in $\mathcal{P}_{\mathrm{GHP}}$, and the Gromov-HausdorffProhoroff space $P_{\mathrm{GHP}}$ is defined by

$$
P_{\mathrm{GHP}}=\mathcal{P}_{\mathrm{GHP}} / \sim,
$$

see, for example, [ADH, p. 4] or [Mie, Section 6.2]. While elements of $P_{\mathrm{GHP}}$ are equivalence classes of measures, we will use the standard convention and identify an equivalence class with its representative, i.e. we will regard the elements of $P_{\mathrm{GHP}}$ as measures and not as equivalence classes of measures.

Next, we define the Gromov-Hausdorff-Prohoroff metric $d_{\mathrm{GHP}}$ on $P_{\mathrm{GHP}}$. For a compact metric space, let $\mathcal{K}(X)$ denote the family of non-empty compact subsets of $X$. For $A, B \in \mathcal{K}(X)$, the Hausdorff distance $d_{\mathrm{H}}(A, B)$ between $A$ and $B$ is defined by

$$
d_{\mathrm{H}}(A, B)=\max \left(\sup _{x \in A} \operatorname{dist}(x, B), \sup _{y \in B} \operatorname{dist}(y, A)\right),
$$

where $\operatorname{dist}(x, E)=\inf _{z \in E} d(x, z)$ for $x \in X$ and $E \subseteq X$. Also, for $\mu, \nu \in \mathcal{P}(X)$, the Prohoroff distance $d_{\mathrm{P}}(\mu, \nu)$ between $\mu$ and $\nu$ is defined as follows. Let $\operatorname{Lip}(X)$ denote the family of Lipschitz functions $f: X \rightarrow \mathbf{R}$ with $|f| \leq 1$ and $\operatorname{Lip}(f) \leq 1$ where $\operatorname{Lip}(f)$ denotes the Lipschitz constant of $f$, i.e. $\operatorname{Lip}(X)=\{f: X \rightarrow \mathbf{R}|| f \mid \leq 1, \operatorname{Lip}(f) \leq 1\}$. The Prohoroff distance $d_{\mathrm{P}}(\mu, \nu)$ between $\mu$ and $\nu$ is defined by

$$
d_{\mathrm{P}}(\mu, \nu)=\sup _{f \in \operatorname{Lip}(X)}\left|\int f d \mu-\int f d \nu\right| .
$$

Finally, the Gromov-Hausdorff-Prohoroff metric $d_{\mathrm{GHP}}$ on $P_{\mathrm{GHP}}$ is defined by

$$
\begin{gathered}
d_{\mathrm{GHP}}(\mu, \nu)=\inf \left\{d_{\mathrm{H}}(f(\operatorname{supp} \mu), g(\operatorname{supp} \nu))+d_{\mathrm{P}}\left(\mu \circ f^{-1}, \nu \circ g^{-1}\right)\right. \\
\mid X \text { is a compact metric space and } f: \operatorname{supp} \mu \rightarrow X \\
\text { and } g: \operatorname{supp} \nu \rightarrow X \text { are isometries }\} .
\end{gathered}
$$

for $\mu, \nu \in P_{\mathrm{GHP}}$, see, for example, [ADH, p. 4] or [Mie, Section 6.2] (see also [Vi]); here, and below, we write $\operatorname{supp} \mu$ for the topological support of a Borel measure $\mu$ on a metric space. The Gromov-Hausdorff-Prohoroff metric extends the Hausdorff metric, the Gromov-Hausdorff metric on the space of all compact metric spaces, and the Prohoroff metric. It can be shown that Gromov-Hausdorff-Prohoroff metric space $\left(P_{\mathrm{GHP}}, d_{\mathrm{GHP}}\right)$ is complete and the classification of "sizes" of subsets of $P_{\mathrm{GHP}}$ using Baire category is therefore meangingful; the reader is referred to [ADH, p. 4] or [Mie, Section 6.2] for a proof of this and for a discussion of the Gromov-HausdorffProhoroff metric space. 
1.2. $\quad L^{q}$-dimensions/Renyi entropies. For a probability measure $\mu$ on a compact metric space, the $L^{q}$-dimensions of $\mu$ are defined as follows, see, for example, [Fal, Pes]; we note that the $L^{q}$-dimensions are also sometimes called the Renyi entropies. For $r>0$ and an extended real number $q$ with $1 \leq q \leq \infty$, write

$$
I_{r}^{q}(\mu)= \begin{cases}\exp \int_{\operatorname{supp} \mu} \log \mu(B(x, r)) d \mu(x) & \text { for } q=1 ; \\ \left(\int_{\operatorname{supp} \mu} \mu(B(x, r))^{q-1} d \mu(x)\right)^{\frac{1}{q-1}} & \text { for } 1<q<\infty \\ \sup _{x \in \operatorname{supp} \mu} \mu(B(x, r)) & \text { for } q=\infty\end{cases}
$$

where $B(x, r)$ denotes the open ball with centre at $x$ and radius equal to $r$. The lower and upper $L^{q}$-dimensions of order $q$ are now defined by

$$
\underline{D}^{q}(\mu)=\liminf _{r \searrow 0} \frac{\log I_{r}^{q}(\mu)}{\log r}, \quad \bar{D}^{q}(\mu)=\limsup _{r \searrow 0} \frac{\log I_{r}^{q}(\mu)}{\log r} .
$$

The definitions of $I_{r}^{1}(\mu)$ and $I_{r}^{\infty}(\mu)$ are, of course, motivated by the fact that $\lim _{q \searrow 1} I_{r}^{q}(\mu)=I_{r}^{1}(\mu)$ and $\lim _{q \nearrow \infty} I_{r}^{q}(\mu)=I_{r}^{\infty}(\mu)$. We also note the following the relationship between the $L^{q}$-dimensions for different values of $q \in[1, \infty]$; below we denote the lower and upper box dimensions of a compact metric space $X$ by $\underline{\operatorname{dim}}_{\mathrm{B}}(X)$ and $\overline{\operatorname{dim}}_{\mathrm{B}}(X)$, respectively (the reader is referred to [Fal] for the definitions of the box dimensions). Namely, for all measures $\mu \in \mathcal{P}(K)$ on a compact metric space $X$ and all $p, q \in(1, \infty)$ with $p \leq q$, we have

$$
\begin{aligned}
& 0 \leq \underline{D}^{\infty}(\mu) \leq \underline{D}^{q}(\mu) \leq \underline{D}^{p}(\mu) \leq \underline{D}^{1}(\mu) \leq \underline{\operatorname{dim}}_{\mathrm{B}}(X), \\
& 0 \leq \bar{D}^{\infty}(\mu) \leq \bar{D}^{q}(\mu) \leq \bar{D}^{p}(\mu) \leq \bar{D}^{1}(\mu) \leq \overline{\operatorname{dim}}_{\mathrm{B}}(X) .
\end{aligned}
$$

Indeed, since $\mu$ is a probability measure, the inequalities $\underline{D}^{\infty}(\mu) \leq \underline{D}^{q}(\mu) \leq \underline{D}^{p}(\mu) \leq$ $\underline{D}^{1}(\mu)$ and $\bar{D}^{\infty}(\mu) \leq \bar{D}^{q}(\mu) \leq \bar{D}^{p}(\mu) \leq \bar{D}^{1}(\mu)$ follow from standard $L^{p}$-norm inequalities, see, for example, [Str, Exercise 6.2.8.(i)]. Finally, the inequalities $\underline{D}^{1}(\mu) \leq$ $\underline{\operatorname{dim}}_{\mathrm{B}}(X)$ and $\bar{D}^{1}(\mu) \leq \overline{\operatorname{dim}}_{\mathrm{B}}(X)$ follow from routine arguments and we have decided to omit the proofs for brevity. In particular, it follows from (1.7) that if $\mu \in P_{\mathrm{GHP}}$ and $p, q \in(1, \infty)$ with $p \leq q$, then

$$
\begin{aligned}
& 0 \leq \underline{D}^{\infty}(\mu) \leq \underline{D}^{q}(\mu) \leq \underline{D}^{p}(\mu) \leq \underline{D}^{1}(\mu) \leq \infty \\
& 0 \leq \bar{D}^{\infty}(\mu) \leq \bar{D}^{q}(\mu) \leq \bar{D}^{p}(\mu) \leq \bar{D}^{1}(\mu) \leq \infty .
\end{aligned}
$$

The $L^{q}$-dimensions were essentially introduced by Rényi [Rén1,Rén2] in 1960 as a tool for analyzing various problems in information theory, and later in the 1980's by theoretical physicists [HJKPS] as a tool for investigating highly irregular distributions. The main significance of the $L^{q}$-dimensions is their relationship with the multifractal spectrum of $\mu$. In the 1980's it was conjectured in the physics literature [HJKPS] that for "good" measures $\mu$ the multifractal spectrum of $\mu$ equals the Legendre transform of the $L^{q}$-dimensions. This result is known as the Multifractal Formalism. During the 1990's there has been an enormous interest in verifying the Multifractal Formalism and computing the multifractal spectra and $L^{q}$-dimensions of measures in the mathematical literature, see [Fal, Pes] and the references therein.

1.3. $L^{q}$-dimensions of typical measures. The purpose of this paper is to investigate the $L^{q}$-dimensions of a typical measure $\mu \in P_{\mathrm{GHP}}$ for $q=1$ and $q=\infty$. We immediately note that the $L^{q}$-dimensions for $1<q<\infty$ of a typical measure have 
been studied earlier [Bay1,Bay2,MR,Ols1,Ols2,Ols3]. For example, Bayart [Bay1], Myjak and Rudnicki [MR] (for $q=2$ ) and Olsen [Ols1] show that if $X$ is a compact Ahlfors regular subset of $\mathbf{R}^{d}$, then a typical measure $\mu \in \mathcal{P}(X)$ satisfies

$$
\begin{array}{ll}
\underline{D}^{q}(\mu)=0 & \text { for all } 1<q<\infty, \\
\bar{D}^{q}(\mu)=\operatorname{dim}_{\mathrm{H}}(X) & \text { for all } 1<q<\infty,
\end{array}
$$

where $\operatorname{dim}_{\mathrm{H}}(X)$ denotes the Hausdorff dimension of $X$. In particular, since the lower box dimension, the upper box dimension and the Hausdorff dimension of a compact Ahlfors regular space $X$ coincide, (1.7) and (1.9) show that a typical measure on $X$ is as "irregular" as possible: the lower dimension $\underline{D}^{q}(\mu)$ is as small as possible and the upper dimension $\bar{D}^{q}(\mu)$ is as big as possible. However, it follows from [Ols3] that by shifting the viewpoint from the study of typical measures belonging to $\mathcal{P}(X)$ for a fixed compact metric space $X$ to the study of typical measures belonging to the "enlarged" space $P_{\mathrm{GHP}}$ of all measures, then the "irregular" behaviour of a typical measure is amplified very dramatically. In particular, the following result follows from [Ols3] and the inequalities in (1.8).

Theorem A. [Ols3, Theorem 1.1] A typical measure $\mu \in P_{\text {GHP satisfies }}$

$$
\begin{aligned}
& \underline{D}^{q}(\mu)=0 \quad \text { for all } 1<q<\infty, \\
& \bar{D}^{q}(\mu)=\infty \quad \text { for all } 1<q<\infty .
\end{aligned}
$$

Proof. This follows almost immediately from [Ols3, Theorem 1.1]. However, since the definitions in [Ols3] differ slightly from the definitions used here, we will provide some further details.

Indeed, in [Ols3] the following slightly different definitions are used. Namely, for $q>1, r>0$ and $\mu \in P_{\mathrm{GHP}}$, let $\mathcal{I}_{r}^{q}(\mu)=\int_{\text {supp } \mu} \mu(B(x, r))^{q-1} d \mu(x)$, and put

$$
\underline{\mathcal{D}}^{q}(\mu)=\liminf _{r \searrow 0} \frac{\log \mathcal{I}_{r}^{q}(\mu)}{-\log r}, \quad \overline{\mathcal{D}}^{q}(\mu)=\limsup _{r \searrow 0} \frac{\log \mathcal{I}_{r}^{q}(\mu)}{-\log r} .
$$

One of the main results in [Ols3, Theorem 1.1] now says that all measures $\mu \in P_{\mathrm{GHP}}$ satisfy $-\infty \leq \underline{\mathcal{D}}^{q}(\mu) \leq \overline{\mathcal{D}}^{q}(\mu) \leq 0$ for all $q>1$, and for a typical measure $\mu \in P_{\mathrm{GHP}}$, we have

$$
\begin{array}{ll}
\underline{\mathcal{D}}^{q}(\mu)=-\infty & \text { for all } q \geq 2, \\
\overline{\mathcal{D}}^{q}(\mu)=0 & \text { for all } q>1 .
\end{array}
$$

However, it is clear that $I_{r}^{q}(\mu)=\mathcal{I}_{r}^{q}(\mu)^{\frac{1}{q-1}}$ for $q>1$, whence

$$
\begin{aligned}
& \underline{D}^{q}(\mu)=\frac{1}{1-q} \overline{\mathcal{D}}^{q}(\mu) \quad \text { for } q>1, \\
& \bar{D}^{q}(\mu)=\frac{1}{1-q} \underline{\mathcal{D}}^{q}(\mu) \quad \text { for } q>1 .
\end{aligned}
$$

We can now prove the statements in Theorem A. To prove the first statement in Theorem A, we note that it follows from (1.11) and (1.12) that for a typical $\mu \in P_{\mathrm{GHP}}$, we have

$$
\underline{D}^{q}(\mu)=\frac{1}{1-q} \overline{\mathcal{D}}^{q}(\mu)=\frac{1}{1-q} 0=0 \quad \text { for all } q>1 .
$$

This proves the first statement in Theorem A. 
To prove the second statement in Theorem A, we note that it follows from (1.10) and (1.13) that for a typical $\mu \in P_{\mathrm{GHP}}$, we have

$$
\bar{D}^{q}(\mu)=\frac{1}{1-q} \mathcal{D}^{q}(\mu)=\frac{1}{1-q}(-\infty)=\infty \quad \text { for all } q \geq 2,
$$

and inequality (1.8) and (1.14) therefore show that for a typical $\mu \in P_{\mathrm{GHP}}$, we have

$$
\bar{D}^{q}(\mu) \geq \bar{D}^{2}(\mu)=\infty \text { for all } 1<q<2 .
$$

The second statement in Theorem A now follows from (1.14) and (1.15).

The first main result shows that the conclusion in Theorem A also holds in the limiting cases for $q=1$ and $q=\infty$. These are the statements in the next theorems.

Theorem 1.1. The case: $q=1$. A typical measure $\mu \in P_{\text {GHP }}$ satisfies

$$
\begin{aligned}
& \underline{D}^{1}(\mu)=0, \\
& \bar{D}^{1}(\mu)=\infty .
\end{aligned}
$$

Theorem 1.2. The case: $q=\infty$. A typical measure $\mu \in P_{G H P}$ satisfies

$$
\begin{aligned}
& \underline{D}^{\infty}(\mu)=0, \\
& \bar{D}^{\infty}(\mu)=\infty .
\end{aligned}
$$

Of course, (1.17) and (1.18) follow immediately from Theorem A and the statements in (1.17) and (1.18) are only included for completeness. The other statements in Theorems 1.1-1.2, namely, (1.16) and (1.19), follow from more general results in Section 1.3. Indeed, Theorems 1.1-1.2 show that the lower $L^{q}$-dimensions for $q=1$ and $q=\infty$ of a typical measure are as small as possible and that the upper $L^{q_{-}}$ dimensions for $q=1$ and $q=\infty$ of a typical measure are as big as possible. Other results, including, for example, (1.9) from [Bay1, Ols1] and Theorem A, as well as the results in [Bay2, Gen, Has, MR, Ols2], investigating the typical $L^{q}$-dimensions and other dimensions of measures show a similar dichotomy. In the next section we will analyse the intriguing dichotomy in Theorem A and Theorems 1.1-1.2 in more detail using the notion of averaging systems. In particular, we will prove more general results about average $L^{q}$-dimensions from which Theorems $1.1-1.2$ are special cases.

1.4. Average $\boldsymbol{L}^{q}$-dimensions of typical measures. For a Borel probability measure $\mu$ with compact support and a real number $q$ with $1 \leq q \leq \infty$, we define the $q^{\prime}$ th moment scaling function $f_{\mu}^{q}:(0, \infty) \rightarrow[0, \infty]$ of $\mu$ by

$$
f_{\mu}^{q}(t)=\frac{\log I_{e^{-t}}^{q}(\mu)}{\log e^{-t}}=\frac{\log I_{e^{-t}}^{q}(\mu)}{-t} .
$$

Using this notation, the $L^{q}$-dimensions of $\mu$ are now given by

$$
\underline{D}^{q}(\mu)=\liminf _{t \rightarrow \infty} f_{\mu}^{q}(t), \quad \bar{D}^{q}(\mu)=\limsup _{t \rightarrow \infty} f_{\mu}^{q}(t),
$$

and Theorem A and Theorems 1.1-1.2 therefore show that the moment scaling function $f_{\mu}^{q}(t)$ of a typical measure $\mu \in P_{\mathrm{GHP}}$ diverges in the worst possible way as $t \rightarrow \infty$. However, there are standard techniques, known as averaging systems, that (at least in some cases) can assign limiting values to divergent functions (the precise definitions will be given below). We will now analysis the divergence of the moment scaling function $f_{\mu}^{q}(t)$ of a typical measure $\mu$ using these ideas. 
We start by recalling the definition of an averaging (or summability) system; the reader is referred to Hardy's classical text [Har] for a systematic treatment of averaging systems.

Definition. Averaging system. An averaging system is a family $\Pi=\left(\Pi_{t}\right)_{t \geq t_{0}}$ with $t_{0}>0$ such that

(i) $\Pi_{t}$ is a finite Borel measure on $\left[t_{0}, \infty\right)$;

(ii) $\Pi_{t}$ has compact support;

(iii) The Consistency Condition: If $f:\left[t_{0}, \infty\right) \rightarrow[0, \infty)$ is a positive measurable function and there is a real number $a$ such that $f(t) \rightarrow a$ as $t \rightarrow \infty$, then $\int f d \Pi_{t} \rightarrow a$ as $t \rightarrow \infty$.

If $f:\left[t_{0}, \infty\right) \rightarrow[0, \infty)$ is a positive measurable function, then we define lower and upper $\Pi$-average of $f$ by

$$
\underline{A}_{\Pi} f=\liminf _{t \rightarrow \infty} \int f d \Pi_{t}
$$

and

$$
\bar{A}_{\Pi} f=\limsup _{t \rightarrow \infty} \int f d \Pi_{t}
$$

respectively.

Applying averaging systems to the moment scaling function $f_{\mu}^{q}(t)$ in (1.20) leads to our key definition, namely, the definition of average $L^{q}$-dimensions.

Definition. Average $L^{q}$-dimension. Let $\Pi=\left(\Pi_{t}\right)_{t \geq t_{0}}$ be an averaging system. Let $q \in[1, \infty]$ and let $\mu$ be a Borel probability measure with compact support. We define the lower and upper $\Pi$-average $L^{q}$-dimensions of $\mu$ by

$$
\underline{D}_{\Pi}^{q}(\mu)=\underline{A}_{\Pi} f_{\mu}^{q}=\liminf _{t \rightarrow \infty} \int \frac{\log I_{e^{-s}}^{q}(\mu)}{-s} d \Pi_{t}(s),
$$

and

respectively.

$$
\bar{D}_{\Pi}^{q}(\mu)=\bar{A}_{\Pi} f_{\mu}^{q}=\limsup _{t \rightarrow \infty} \int \frac{\log I_{e^{-s}}^{q}(\mu)}{-s} d \Pi_{t}(s),
$$

We first note that it follows by an argument similar to the proof of (1.8) that the average $L^{q}$-dimensions satisfy the following inequalities for $1<p \leq q<\infty$, namely, we have

$$
\begin{aligned}
& 0 \leq \underline{D}_{\Pi}^{\infty}(\mu) \leq \underline{D}_{\Pi}^{q}(\mu) \leq \underline{D}_{\Pi}^{p}(\mu) \leq \underline{D}_{\Pi}^{1}(\mu) \leq \infty, \\
& 0 \leq \bar{D}_{\Pi}^{\infty}(\mu) \leq \bar{D}_{\Pi}^{q}(\mu) \leq \bar{D}_{\Pi}^{p}(\mu) \leq \bar{D}_{\Pi}^{1}(\mu) \leq \infty .
\end{aligned}
$$

We also note that $L^{q}$-dimensions are, in fact, average $L^{q}$-dimensions. Indeed, if $\mu$ is a Borel probability measure with compact support and we let $\Pi$ denote the average system defined by $\Pi=\left(\delta_{t}\right)_{t \geq 1}$ (where $\delta_{t}$ denotes the Dirac measure concentrated at $t$ ), then clearly

$$
\underline{D}_{\Pi}^{q}(\mu)=\underline{D}^{q}(\mu), \quad \bar{D}_{\Pi}^{q}(\mu)=\bar{D}^{q}(\mu) .
$$

In [Ols3] is was shown that not only is the moment scaling function $f_{\mu}^{q}(t)$ for $1<q<\infty$ of a typical measure $\mu$ divergent as $t \rightarrow \infty$, but it is so irregular that it remains spectacularly divergent as $t \rightarrow \infty$ even after being "averaged" or "smoothened out" using arbitrarily powerful averaging systems; this is the contents of Theorem B below and follows from [Ols3, Theorem 1.4] and the inequalities in (1.21) by an argument similar to the proof of Theorem A. 
Theorem B. [Ols3, Theorem 1.4] Let $\Pi$ be an averaging system. A typical measure $\mu \in P_{\text {GHP }}$ satisfies

$$
\begin{aligned}
& \underline{D}_{\Pi}^{q}(\mu)=0 \quad \text { for all } 1<q<\infty, \\
& \bar{D}_{\Pi}^{q}(\mu)=\infty \quad \text { for all } 1<q<\infty .
\end{aligned}
$$

The second main result shows that the conclusion in Theorem B also holds in the limiting cases for $q=1$ and $q=\infty$. These are the statements in the next theorems.

Theorem 1.3. The case: $q=1$. Let $\Pi$ be an averaging system. A typical measure $\mu \in P_{\text {GHP }}$ satisfies

$$
\begin{aligned}
& \underline{D}_{\Pi}^{1}(\mu)=0, \\
& \bar{D}_{\Pi}^{1}(\mu)=\infty .
\end{aligned}
$$

Theorem 1.4. The case: $q=\infty$. Let $\Pi$ be an averaging system. A typical measure $\mu \in P_{G H P}$ satisfies

$$
\begin{aligned}
& \underline{D}_{\Pi}^{\infty}(\mu)=0, \\
& \bar{D}_{\Pi}^{\infty}(\mu)=\infty .
\end{aligned}
$$

Of course, (1.24) and (1.25) follow immediately from Theorem B and the statements in (1.24) and (1.25) are only included for completeness. The proofs of the other statements in Theorems 1.3-1.4, namely, (1.23) and (1.26), are given in Sections 3-6. Sections 3-4 contains various semi-continuity results that may be of interest in their own right; the reader is referred to Theorems 1.5-1.6 in Section 1.5 for a detailed discussion of this. The proof of (1.23) is given in Section 5 and the proof of (1.26) is given in Section 6. We now make some remarks about the proofs and statements in Theorems 1.3-1.4.

Remark. We note that the statement in Theorem B follows immediately from Theorems 1.3-1.4 and the inequalities in (1.21), and Theorems 1.3-1.4 therefore provide an alternative proof of Theorem B.

Remark. Observe that if we apply Theorem 1.3 and Theorem 1.4 to the averaging system $\Pi$ defined by $\Pi=\left(\delta_{t}\right)_{t \geq 1}$, then it follows from (1.22) that the statements in Theorem 1.3 and Theorem 1.4 reduce to the statements in Theorem 1.1 and Theorem 1.2 , respectively.

1.5. Semi-continuity of $\boldsymbol{L}^{q}$-dimensions/Renyi entropies. The proofs of Theorem 1.3 and Theorem 1.4 rely on two semi-continuity results of the $L^{q_{-}}$ dimensions/Renyi entropies for $q=1$ and $q=\infty$. Continuity and semi-continuity properties of entropies form a fundamental and important part of ergodic theory and information theory (see, for example, [Wa, Chapter 8]), and these results may therefore be of interest in their own right. For this reason we have decided to state the results explicitly. We first recall and define the following notation. Recall, that the $r$-approximative $L^{q}$-dimension/Renyi entropy $I_{r}^{1}: P_{\mathrm{GHP}} \rightarrow \mathbf{R}$ for $q=1$ is defined by

$$
I_{r}^{1}(\mu)=\exp \int_{\operatorname{supp} \mu} \log \mu(B(x, r)) d \mu(x)
$$

Also for a compact metric space $X$ and $x \in X$, we let $C(x, r)$ denote the closed ball with centre at $x$ and radius equal to $r$, i.e. $C(x, r)=\{y \in X \mid d(x, y) \leq r\}$, and define 
$J_{r}^{1}: P_{\mathrm{GHP}} \rightarrow \mathbf{R}$ by

$$
J_{r}^{1}(\mu)=\exp \int_{\operatorname{supp} \mu} \log \mu(C(x, r)) d \mu(x) .
$$

Next, recall that that the $r$-approximative $L^{q}$-dimension/Renyi entropy $I_{r}^{\infty}: P_{\mathrm{GHP}} \rightarrow$ $\mathbf{R}$ for $q=\infty$ is defined by

$$
I_{r}^{\infty}(\mu)=\sup _{x \in \operatorname{supp} \mu} \mu(B(x, r))
$$

and define $J_{r}^{\infty}: P_{\mathrm{GHP}} \rightarrow \mathbf{R}$ by

$$
J_{r}^{\infty}(\mu)=\sup _{x \in \operatorname{supp} \mu} \mu(C(x, r)) .
$$

We can now state the semi-continuity results that the proofs of Theorem 1.3 and Theorem 1.4 rely on; this is done in Theorem 1.5 and Theorem 1.6 below. We provide a detailed discussion of Theorem 1.5 and Theorem 1.6 after we have stated them.

\section{Theorem 1.5.}

(1) The function $I_{r}^{1}: P_{G H P} \rightarrow \mathbf{R}$ is lower semi-continuous on $P_{G H P}$ for all $r>0$.

(2) The function $J_{r}^{1}: P_{G H P} \rightarrow \mathbf{R}$ is upper semi-continuous on $P_{G H P}$ for all $r>0$.

\section{Theorem 1.6.}

(1) The function $I_{r}^{\infty}: P_{G H P} \rightarrow \mathbf{R}$ is lower semi-continuous on $P_{G H P}$ for all $r>0$.

(2) The function $J_{r}^{\infty}: P_{G H P} \rightarrow \mathbf{R}$ is upper semi-continuous on $P_{G H P}$ for all $r>0$.

The proof of Theorem 1.5 is given in Section 3 and the proof of Theorem 1.6 is given in Section 4. The proof of Theorem 1.3 relies crucially on Theorem 1.5, and the proof of Theorem 1.4 relies crucially on Theorem 1.6. While it is not very difficult to show that the functions $I_{r}^{\infty}$ and $J_{r}^{\infty}$ are semi-continuous, we found it far more challenging to show that the functions $I_{r}^{1}$ and $J_{r}^{1}$ are semi-continuous. For example, in order to show that $I_{r}^{1}$ is lower semi-continuous, we must show that if $\left(\mu_{n}\right)_{n}$ is a sequence in $P_{\mathrm{GHP}}$ and $\mu \in P_{\mathrm{GHP}}$ such that $\mu_{n} \rightarrow \mu$ with respect to $d_{\mathrm{GHP}}$, then

$$
I_{r}^{1}(\mu) \leq \liminf _{n} I_{r}^{1}\left(\mu_{n}\right),
$$

i.e. (using the fact that the exponential function is continuous and increasing) we must prove that

$$
\int_{\operatorname{supp} \mu} \log \mu(B(x, r)) d \mu(x) \leq \liminf _{n} \int_{\operatorname{supp} \mu} \log \mu_{n}(B(x, r)) d \mu_{n}(x) .
$$

Since $\mu_{m} \rightarrow \mu$ weakly (because $\mu_{m} \rightarrow \mu$ with respect to $d_{\mathrm{GHP}}$ ), we conclude that $\log \mu(B(x, r)) \leq \liminf _{m} \log \mu_{m}(B(x, r))$, whence (using Fatou's lemma)

$$
\begin{aligned}
\int_{\operatorname{supp} \mu} \log \mu(B(x, r)) d \mu(x) & \leq \int_{\operatorname{supp} \mu} \liminf _{m} \log \mu_{m}(B(x, r)) d \mu(x) \\
& \leq \liminf _{m} \int_{\operatorname{supp} \mu} \log \mu_{m}(B(x, r)) d \mu(x) .
\end{aligned}
$$

Also, since $\mu_{n} \rightarrow \mu$ weakly and since it is not difficult to see that the function $x \rightarrow \log \mu_{m}(B(x, r))$ is lower semi-continuous for each $m$, we conclude that for each fixed $m$, we have

$$
\int_{\operatorname{supp} \mu} \log \mu_{m}(B(x, r)) d \mu(x) \leq \liminf _{n} \int_{\operatorname{supp} \mu} \log \mu_{m}(B(x, r)) d \mu_{n}(x) .
$$


Combining (1.28) and (1.29) shows that

$$
\int_{\operatorname{supp} \mu} \log \mu(B(x, r)) d \mu(x) \leq \liminf _{m} \liminf _{n} \int_{\operatorname{supp} \mu} \log \mu_{m}(B(x, r)) d \mu_{n}(x) .
$$

Of course, inequality (1.30) is not the same as inequality (1.27). Indeed, in (1.30), the indices $n$ and $m$ tend to infinity independently whereas this is not the case in (1.27). Unfortunately, we found it surprisingly difficult to overcome this problem, and the proof of the lower semi-continuity of the function $I_{r}^{1}: P_{\mathrm{GHP}} \rightarrow \mathbf{R}$ is therefore considerably more involved using the power-series expansion of the logarithmic function to show that $\log I_{r}^{1}$ can be written as a converging series of lower semi-continuous functions. Finally, since we can show that this series converges sufficiently rapidly, this allow is us to conclude that $I_{r}^{1}$ is lower semi-continuous. The reader is referred to Section 4 for the details of this argument.

As an application of Theorems 1.3-1.4 we will now consider higher order Hölder and Cesàro averages of the moment scaling function $f_{\mu}^{q}$ of a typical measure $\mu$; this is done in next section.

\section{Hölder and Cesàro averages of the $L^{q}$-dimension of a typical measure}

Two of the most commonly used averaging systems are Hölder averages and Cesàro averages. We will now define these averaging systems and apply them to the moment scaling function $f_{\mu}^{q}(t)=\frac{\log I_{e-t}(\mu)}{t}$ of a Borel probability measure $\mu$. For $a>0$ and a positive measurable function $f:(a, \infty) \rightarrow[0, \infty)$, we define $M f:(a, \infty) \rightarrow$ $[0, \infty)$ by

$$
(M f)(t)=\frac{1}{t} \int_{a}^{t} f(s) d s .
$$

For a positive integer $n$, we now define the lower and upper $n$ 'th order Hölder averages of $f$ by

$$
\underline{H}_{n} f=\liminf _{t \rightarrow \infty}\left(M^{n} f\right)(t), \quad \bar{H}_{n} f=\limsup _{t \rightarrow \infty}\left(M^{n} f\right)(t) .
$$

The Cesàro averages are defined as follows. First, we define If $:(a, \infty) \rightarrow[0, \infty)$ by

$$
(I f)(t)=\int_{a}^{t} f(s) d s
$$

For a positive integer $n$, we now define the lower and upper $n$ 'th order Cesàro averages of $f$ by

$$
\underline{C}_{n} f=\liminf _{t \rightarrow \infty} \frac{n !}{t^{n}}\left(I^{n} f\right)(t), \quad \bar{C}_{n} f=\limsup _{t \rightarrow \infty} \frac{n !}{t^{n}}\left(I^{n} f\right)(t) .
$$

It is well-known that that the Hölder and Cesàro averages satisfy the following inequalities, namely,

$$
\begin{aligned}
& \liminf _{t \rightarrow \infty} f(t)=\underline{H}_{0} f \leq \underline{H}_{1} f \leq \underline{H}_{2} f \leq \ldots \leq \bar{H}_{2} f \leq \bar{H}_{1} f \leq \bar{H}_{0} f=\limsup _{t \rightarrow \infty} f(t), \\
& \liminf _{t \rightarrow \infty} f(t)=\underline{C}_{0} f \leq \underline{C}_{1} f \leq \underline{C}_{2} f \leq \ldots \leq \bar{C}_{2} f \leq \bar{C}_{1} f \leq \bar{C}_{0} f=\limsup _{t \rightarrow \infty} f(t) .
\end{aligned}
$$

It is also well-known that the Hölder and Cesàro averages can be expressed using averaging systems in the sense of the definition in Section 1.4. Indeed, if for a positive integer $n$, we define the averaging system $\Pi_{n}^{\mathrm{H}}=\left(\Pi_{n, t}^{\mathrm{H}}\right)_{t \geq a}$ by

$$
\Pi_{n, t}^{\mathrm{H}}(B)=\frac{1}{(n-1) ! t} \int_{[a, t] \cap B}(\log t-\log s)^{n-1} d s
$$


for Borel subsets $B$ of $[a, \infty)$, then

$$
\underline{H}_{n} f=\liminf _{t} \int f d \Pi_{n, t}^{\mathrm{H}}, \quad \bar{H}_{n} f=\limsup _{t} \int f d \Pi_{n, t}^{\mathrm{H}},
$$

see, for example, [Ja, p. 675]. Similarly, if for a positive integer $n$, we define the averaging system $\Pi_{n}^{\mathrm{C}}=\left(\Pi_{n, t}^{\mathrm{C}}\right)_{t \geq a}$ by

$$
\Pi_{n, t}^{\mathrm{C}}(B)=\frac{n}{t^{n}} \int_{[a, t] \cap B}(t-s)^{n-1} d s
$$

then

$$
\underline{C}_{n} f=\liminf _{t} \int f d \Pi_{n, t}^{\mathrm{C}}, \quad \bar{C}_{n} f=\limsup _{t} \int f d \Pi_{n, t}^{\mathrm{C}},
$$

see, for example, [Har, pp. 110-111].

Using Hölder and Cesàro averages we can now introduce average Hölder and Cesàro $L^{q}$-dimensions by applying the definitions of the Hölder and Cesàro averages to the function $f_{\mu}^{q}(t)$. This is the content of the next definition.

Definition. Average Hölder and Cesàro $L^{q}$-dimensions. Let $\mu \in P_{\mathrm{GHP}}$. For $q \in[1, \infty]$, we define the lower and upper $n$ 'th order average Hölder $L^{q}$-dimension of $\mu$, denoted by $\underline{D}_{\mathrm{H}, n}^{q}(\mu)$ and $\bar{D}_{\mathrm{H}, n}^{q}(\mu)$, as the lower and upper $n$ 'th order Hölder average of the function $f_{\mu}^{q}(t)$ for $t \geq 1$, i.e. we put

$$
\underline{D}_{\mathrm{H}, n}^{q}(\mu)=\underline{H}_{n} f_{\mu}^{q}, \quad \bar{D}_{\mathrm{H}, n}^{q}(\mu)=\bar{H}_{n} f_{\mu}^{q} .
$$

Similarly, we define the lower and upper $n$ 'th order average Cesàro $L^{q}$-dimension of $\mu$, denoted by $\underline{D}_{\mathrm{C}, n}^{q}(\mu)$ and $\bar{D}_{\mathrm{C}, n}^{q}(\mu)$, by

$$
\underline{D}_{\mathrm{C}, n}^{q}(\mu)=\underline{C}_{n} f_{\mu}^{q}, \quad \bar{D}_{\mathrm{C}, n}^{q}(\mu)=\bar{C}_{n} f_{\mu}^{q} .
$$

The higher order average Hölder and Cesàro $L^{q}$-dimensions form a double infinite hierarchy in (at least) countably infinite many levels, namely, we have (using (2.1))

$$
\begin{aligned}
& \underline{D}^{q}(\mu)=\underline{D}_{\mathrm{H}, 0}^{q}(\mu) \leq \underline{D}_{\mathrm{H}, 1}^{q}(\mu) \leq \ldots \leq \bar{D}_{\mathrm{H}, 1}^{q}(\mu) \leq \bar{D}_{\mathrm{H}, 0}^{q}(\mu)=\bar{D}^{q}(\mu), \\
& \underline{D}^{q}(\mu)=\underline{D}_{\mathrm{C}, 0}^{q}(\mu) \leq \underline{D}_{\mathrm{C}, 1}^{q}(\mu) \leq \ldots \leq \bar{D}_{\mathrm{C}, 1}^{q}(\mu) \leq \bar{D}_{\mathrm{C}, 0}^{q}(\mu)=\bar{D}^{q}(\mu) .
\end{aligned}
$$

As an application of Theorem B and Theorems 1.3-1.4, we will now show that the behaviour of a typical measure $\mu \in P_{\mathrm{GHP}}$ is so irregular that not even the hierarchies in (2.2) formed by taking Hölder and Cesàro averages of all orders are sufficiently powerful to "smoothen out" the behaviour of the box counting function $f_{\mu}^{q}(t)$ for $q \in[1, \infty]$ as $t \rightarrow \infty$.

Theorem 2.1. A typical measure $\mu \in P_{G H P}$ satisfies

$$
\begin{aligned}
& \underline{D}_{\mathrm{H}, n}^{q}(\mu)=\underline{D}_{\mathrm{C}, n}^{q}(\mu)=0 \quad \text { for all } 1 \leq q \leq \infty, \\
& \bar{D}_{\mathrm{H}, n}^{q}(\mu)=\bar{D}_{\mathrm{C}, n}^{q}(\mu)=\infty \quad \text { for all } 1 \leq q \leq \infty,
\end{aligned}
$$

for all $n \in \mathbf{N} \cup\{0\}$.

Proof. This statement follows immediately from Theorem B and Theorems 1.31.4 . 


\section{Proof of Theorem 1.6}

Fix $\mu \in P_{\mathrm{GHP}}$. Recall, that for $r>0$, we write

$$
I_{r}^{\infty}(\mu)=\sup _{x \in \operatorname{supp} \mu} \mu(B(x, r)) .
$$

Also recall that for a compact metric space $X$ and $x \in X$, we let $C(x, r)$ denote the closed ball with centre at $x$ and radius equal to $r$, i.e. $C(x, r)=\{y \in X \mid d(x, y) \leq r\}$, and write

$$
J_{r}^{\infty}(\mu)=\sup _{x \in \operatorname{supp} \mu} \mu(C(x, r)) .
$$

We also introduce the dual notions, namely, for $r>0$, we write

$$
I_{r}^{-\infty}(\mu)=\inf _{x \in \operatorname{supp} \mu} \mu(B(x, r)),
$$

and

$$
J_{r}^{-\infty}(\mu)=\inf _{x \in \operatorname{supp} \mu} \mu(C(x, r)) .
$$

The purpose of this section is twofold. The first purpose is to state and prove Proposition 3.2, saying that the functions $I_{r}^{\infty}$ and $J_{r}^{\infty}$ are semi-continuous, and Proposition 3.3, saying that the the functions $I_{r}^{-\infty}$ and $J_{r}^{-\infty}$ are semi-continuous, and the second purpose is to prove Theorem 1.6. The semi-continuity results in Propositions 3.2-3.3 play crucial parts in the proofs of Theorems 1.3-1.5 in Sections $4-6$ but may also be of interest in their own right. We start by proving the following lemma.

Lemma 3.1. Let $X$ be a metric space. Let $C \in \mathcal{K}(X)$ and for each positive integer $n$, let $C_{n} \in \mathcal{K}(X)$. Let $x \in X$ and for each positive integer $n$, let $x_{n} \in C_{n}$. Assume that $C_{n} \rightarrow C$ with respect to $d_{H}$ and that $x_{n} \rightarrow x$. Then $x \in C$.

Proof. Assume, in order to reach a contradiction, that $x \notin C$. We can therefore find a positive real number $r>0$ such that $B(x, r) \subseteq X \backslash C$. Also, since $x_{n} \rightarrow x$, we can choose a positive integer $N$ such that $x_{n} \in B\left(x, \frac{r}{2}\right)$ for all $n \geq N$. In particular, we conclude that $C \cap B(x, r)=\varnothing$ and $C_{n} \cap B\left(x, \frac{r}{2}\right) \neq \varnothing$ for $n \geq N$, and so $d_{\mathrm{H}}\left(C_{n}, C\right) \geq \frac{r}{2}$ for all $n \geq N$. However, this contradicts the fact that $C_{n} \rightarrow C$ with respect to $d_{\mathrm{H}}$.

We can now state the first main semi-continuity results in the section.

\section{Proposition 3.2.}

(1) The function $I_{r}^{\infty}: P_{G H P} \rightarrow \mathbf{R}$ is lower semi-continuous on $P_{G H P}$ for all $r>0$.

(2) The function $J_{r}^{\infty}: P_{G H P} \rightarrow \mathbf{R}$ is upper semi-continuous on $P_{G H P}$ for all $r>0$.

Proof. (2) Let $\mu \in P_{\mathrm{GHP}}$ and let $\mu_{n} \in P_{\mathrm{GHP}}$ for $n \in \mathbf{N}$ with $\mu_{n} \rightarrow \mu$ with respect to $d_{\mathrm{GHP}}$. For brevity write $\Lambda=\lim \sup _{n} J_{r}^{\infty}\left(\mu_{n}\right)$ and note that we must now prove that $\Lambda \leq J_{r}^{\infty}(\mu)$.

We may clearly assume that there is a compact metric space $X$ such that $\mu \in$ $\mathcal{P}(X)$ and $\mu_{n} \in \mathcal{P}(X)$ for $n \in \mathbf{N}$.

We can clearly find a subsequence $\left(\mu_{n_{k}}\right)_{k}$ such that

$$
J_{r}^{\infty}\left(\mu_{n_{k}}\right) \rightarrow \Lambda \text {. }
$$

Next, fix $\varepsilon>0$, and note that for each positive integer $n$, we can choose a point $x_{n} \in \operatorname{supp} \mu_{n}$ such that

$$
J_{r}^{\infty}\left(\mu_{n}\right)-\varepsilon \leq \mu_{n}\left(C\left(x_{n}, r\right)\right)
$$


It also follows from the compactness of $X$ that there is a subsequence $\left(x_{n_{k_{l}}}\right)_{l}$ and a point $x_{0} \in X$ such that

$$
x_{n k_{l}} \rightarrow x_{0} .
$$

Since $x_{n k_{l}} \rightarrow x_{0}$, it follows that we can find a positive integer $L_{\varepsilon}$ such that if $l \geq L_{\varepsilon}$, then

$$
C\left(x_{n_{k_{l}}}, r\right) \subseteq C\left(x_{0}, r+\varepsilon\right) .
$$

In particular, we conclude from this that

$$
\limsup _{l} \mu_{n_{k_{l}}}\left(C\left(x_{n_{k_{l}}}, r\right)\right) \leq \limsup _{l} \mu_{n_{k_{l}}}\left(C\left(x_{0}, r+\varepsilon\right)\right) .
$$

However, since $\mu_{n} \rightarrow \mu$ with respect to $d_{\mathrm{GHP}}$, it follows that $\mu_{n} \rightarrow \mu$ with respect to $d_{\mathrm{P}}$, i.e. $\mu_{n} \rightarrow \mu$ with respect to the weak topology, and so $\mu_{n_{k_{l}}} \rightarrow \mu$ with respect to the weak topology, It follows immediately from this and the fact that the set $C\left(x_{0}, r+\varepsilon\right)$ is closed that

$$
\limsup _{l} \mu_{n_{k_{l}}}\left(C\left(x_{0}, r+\varepsilon\right)\right) \leq \mu\left(C\left(x_{0}, r+\varepsilon\right)\right) .
$$

Combining (3.3) and (3.4) now shows that

$$
\limsup _{l} \mu_{n_{k_{l}}}\left(C\left(x_{n_{k_{l}}}, r\right)\right) \leq \mu\left(C\left(x_{0}, r+\varepsilon\right)\right) .
$$

Hence, using (3.1), (3.2) and (3.5),

$$
\begin{aligned}
\Lambda & =\lim _{k} J_{r}^{\infty}\left(\mu_{n_{k}}\right)=\lim _{l} J_{r}^{\infty}\left(\mu_{n_{k_{l}}}\right) \\
& \leq \limsup _{l}\left(\mu_{n_{k_{l}}}\left(C\left(n_{k_{l}}, r\right)\right)+\varepsilon\right) \leq \mu\left(C\left(x_{0}, r+\varepsilon\right)\right)+\varepsilon
\end{aligned}
$$

for all $\varepsilon$. Since (3.6) holds for all $\varepsilon>0$, it follows that

$$
\begin{aligned}
\Lambda & \leq \inf _{\varepsilon>0}\left(\mu\left(C\left(x_{0}, r+\varepsilon\right)\right)+\varepsilon\right) \\
& =\inf _{\varepsilon>0} \mu\left(C\left(x_{0}, r+\varepsilon\right)\right)=\mu\left(\bigcap_{\varepsilon>0} C\left(x_{0}, r+\varepsilon\right)\right) .
\end{aligned}
$$

However, since clearly $\bigcap_{\varepsilon>0} C\left(x_{0}, r+\varepsilon\right)=C\left(x_{0}, r\right)$, we deduce from (3.7) that

$$
\Lambda \leq \mu\left(C\left(x_{0}, r\right)\right) .
$$

Finally, note that since $\mu_{n_{k}} \rightarrow \mu$ with respect to $d_{\mathrm{GHP}}$, it follows that $\operatorname{supp} \mu_{n_{k}} \rightarrow$ $\operatorname{supp} \mu$ with respect to $d_{\mathrm{H}}$. Since also $x_{n_{k}} \in \operatorname{supp} \mu_{n_{k}}$ and $x_{n_{k}} \rightarrow x_{0}$, we deduce from this and Lemma 3.1 that $x_{0} \in \operatorname{supp} \mu$, whence

$$
\mu\left(C\left(x_{0}, r\right)\right) \leq \sup _{x \in \operatorname{supp} \mu} \mu(C(x, r))=J_{r}^{\infty}(\mu) .
$$

The desired result now follows from (3.8) and (3.9).

(1) The proof of Part (1) is similar to the proof of Part (2), and for sake of brevity we have therefore decided to omit it.

We now state the second main result in this section.

Proposition 3.3.

(1) The function $I_{r}^{-\infty}: P_{G H P} \rightarrow \mathbf{R}$ is lower semi-continuous on $P_{G H P}$ for all $r>0$.

(2) The function $J_{r}^{-\infty}: P_{G H P} \rightarrow \mathbf{R}$ is upper semi-continuous on $P_{G H P}$ for all $r>0$. 
Proof. The proof of this proposition is very similar to the proof of Proposition 3.2, and for the sake of brevity we have therefore decided to omit the proof.

Finally, we prove Theorem 1.6.

Proof of Theorem 1.6. This result follows immediately from Proposition 3.2.

\section{Proof of Theorem 1.5.}

Recall that for $r>0$, the functions $I_{r}^{1}, J_{r}^{1}: P_{\mathrm{GHP}} \rightarrow \mathbf{R}$ are defined by

$$
\begin{aligned}
& I_{r}^{1}(\mu)=\exp \int_{\operatorname{supp} \mu} \log \mu(B(x, r)) d \mu(x), \\
& J_{r}^{1}(\mu)=\exp \int_{\operatorname{supp} \mu} \log \mu(C(x, r)) d \mu(x) ;
\end{aligned}
$$

see Section 1.5. The purpose of this section is to prove Theorem 1.5 saying that the functions $I_{r}^{1}$ and $J_{r}^{1}$ are semi-continuous. Fix $r>0$. Define $f, g: P_{\mathrm{GHP}} \rightarrow \mathbf{R}$ by

$$
\begin{aligned}
& f(\mu)=\int_{\operatorname{supp} \mu} \log \mu(B(x, r)) d \mu(x), \\
& g(\mu)=\int_{\operatorname{supp} \mu} \log \mu(C(x, r)) d \mu(x),
\end{aligned}
$$

and for a positive integer $m$, define $f_{m}, g_{m}: P_{\mathrm{GHP}} \rightarrow \mathbf{R}$ by

$$
\begin{aligned}
& f_{m}(\mu)=\int_{\operatorname{supp} \mu} \mu(\operatorname{supp} \mu \backslash B(x, r))^{m} d \mu(x), \\
& g_{m}(\mu)=\int_{\operatorname{supp} \mu} \mu(\operatorname{supp} \mu \backslash C(x, r))^{m} d \mu(x) .
\end{aligned}
$$

Before proving Theorem 1.5, we first prove the following six auxiliary lemmas.

Lemma 4.1. For integers $n, m \in \mathbf{N}$, let $x_{m, n} \geq 0$.

(1) If

$$
\limsup _{n} \sum_{m \geq M} x_{m, n} \rightarrow 0 \quad \text { as } M \rightarrow \infty
$$

then we have

$$
\limsup _{n} \sum_{m \geq 1} x_{m, n} \leq \sum_{m \geq 1} \limsup _{n} x_{m, n}
$$

(2) We have

$$
\liminf _{n} \sum_{m \geq 1} x_{m, n} \geq \sum_{m \geq 1} \liminf _{n} x_{m, n}
$$

Remark. Before proving Lemma 4.1 we make the following remark. While the statement in Lemma 4.1.(2) is a trivial consequence of Fatou's Lemma, the "reverse" statement in Lemma 4.1.(1) does not follow from the Reverse Fatou's Lemma; recall that the Reverse Fatou's Lemma says the following: if $(M, \mathcal{E}, \mu)$ is a measure space and $\left(\varphi_{n}\right)_{n}$ is a sequence of positive measurable functions $\varphi_{n}: M \rightarrow[0, \infty]$ with $\int \sup _{n} \varphi_{n} d \mu<\infty$, then $\lim \sup _{n} \int \varphi_{n} d \mu \leq \int \lim \sup _{n} \varphi_{n} d \mu$. We will now explain this in more detail. Indeed, consider the following two conditions:

$$
\begin{aligned}
& \text { Condition }(*): \sum_{m \geq 1} \sup _{n} x_{m, n}<\infty, \\
& \text { Condition }(* *): \lim \sup _{n} \sum_{m \geq M} x_{m, n} \rightarrow 0 \text { as } M \rightarrow \infty,
\end{aligned}
$$


and note that Condition $(* *)$ is weaker than Condition $(*)$, i.e.

$$
\text { Condition }(*) \Longrightarrow \text { Condition }(* *) \text {; }
$$

indeed, in order to prove this implication we note that Condition $(*)$ implies that $\sum_{m \geq M} \sup _{k} x_{m, k} \rightarrow 0$ as $M \rightarrow \infty$, whence

$$
\limsup _{n} \sum_{m \geq M} x_{m, n} \leq \limsup _{n} \sum_{m \geq M} \sup _{k} x_{m, k}=\sum_{m \geq M} \sup _{k} x_{m, k} \rightarrow 0
$$

as $M \rightarrow \infty$, and Condition $(* *)$ is therefore satisfied.

If Condition $(*)$ is satisfied, then it follows immediately from the Reverse Fatou's Lemma that

$$
\limsup _{n} \sum_{m \geq 1} x_{m, n} \leq \sum_{m \geq 1} \limsup _{n} x_{m, n} .
$$

Unfortunately, we have not been able to verify that Condition $(*)$ is satisfied for the particular choice of $x_{m, n}$ that we need. In stead, we can show that the weaker Condition $(* *)$ is satisfied for the choice of $x_{m, n}$ that we need, and Lemma 4.1.(1) says that the weaker Condition $(* *)$ is, in fact, sufficient to guarantee that the conclusion of the Reverse Fatou's Lemma is valid, i.e. it is sufficient to guarantee that we have

$$
\limsup _{n} \sum_{m \geq 1} x_{m, n} \leq \sum_{m \geq 1} \limsup _{n} x_{m, n} .
$$

Proof of Lemma 4.1. (1) Since limsup is finitely sub-additive, it follows that if $M$ is a positive integer, then

$$
\limsup _{n} \sum_{m \geq 1} x_{m, n} \leq \sum_{m<M} \limsup _{n} x_{m, n}+\limsup _{n} \sum_{m \geq M} x_{m, n}
$$

By letting $M \rightarrow \infty$ in the previous inequality, we now conclude that

$$
\limsup _{n} \sum_{m \geq 1} x_{m, n} \leq \sum_{m \geq 1} \limsup _{n} x_{m, n}+\limsup _{M} \limsup _{n} \sum_{m \geq M} x_{m, n} .
$$

The desired result follows immediately from assumption (4.1) and inequality (4.3).

(2) This statement follows immediately from Fatou's Lemma.

Lemma 4.2. Let $\mu \in P_{G H P}$ and define $\varphi: \operatorname{supp} \mu \rightarrow \mathbf{R}$ by $\varphi(x)=\mu(B(x, r))$.

(1) The function $\varphi: \operatorname{supp} \mu \rightarrow \mathbf{R}$ is lower semi-continuous.

(2) $I_{r}^{-\infty}(\mu)>0$; recall that the function $I_{r}^{-\infty}: P_{G H P} \rightarrow \mathbf{R}$ is defined at the beginning of Section 3.

Proof. (1) Let $\left(x_{n}\right)_{n}$ be a sequence in $\operatorname{supp} \mu$ and let $x \in \operatorname{supp} \mu$ and assume that $x_{n} \rightarrow x$. We must now prove that $\varphi(x) \leq \liminf _{n} \varphi\left(x_{n}\right)$. Fix $\varepsilon$. Since $x_{n} \rightarrow x$, we can choose a positive integer $N_{\varepsilon}$ such that if $n \geq N_{\varepsilon}$, then $B(x, r-\varepsilon) \subseteq B\left(x_{n}, r\right)$, and so $\mu(B(x, r-\varepsilon)) \leq \mu\left(B\left(x_{n}, r\right)\right)=\varphi\left(x_{n}\right)$ for all $n \geq N_{\varepsilon}$. This clearly implies that $\mu(B(x, r-\varepsilon)) \leq \liminf _{n} \varphi\left(x_{n}\right)$. Since $\varepsilon>0$ was arbitrary, we conclude from this that

$$
\sup _{\varepsilon>0} \mu(B(x, r-\varepsilon)) \leq \liminf _{n} \varphi\left(x_{n}\right) .
$$

However, as $\cup_{\varepsilon>0} B(x, r-\varepsilon)=B(x, r)$, we have $\sup _{\varepsilon>0} \mu(B(x, r-\varepsilon))=\mu\left(\cup_{\varepsilon>0} B(x, r-\right.$ $\varepsilon))=\mu(B(x, r))=\varphi(x)$, and it therefore follows immediately from (4.4) that $\varphi(x) \leq$ $\liminf \operatorname{in}_{n} \varphi\left(x_{n}\right)$.

(2) Indeed, since $\operatorname{supp} \mu$ is compact and since it follows from (1) that $\varphi: \operatorname{supp} \mu \rightarrow$ $\mathbf{R}$ is lower semi-continuous, it is well-known that there is a point $x_{0} \in \operatorname{supp} \mu$ such 
that $\varphi\left(x_{0}\right)=\inf _{x \in \operatorname{supp} \mu} \varphi(x)$. This implies that $I_{r}^{-\infty}(\mu)=\inf _{x \in \operatorname{supp} \mu} \mu(B(x, r))=$ $\inf _{x \in \operatorname{supp} \mu} \varphi(x)=\varphi\left(x_{0}\right)=\mu\left(B\left(x_{0}, r\right)\right)$. However, since $x_{0} \in \operatorname{supp} \mu$, we have $\mu\left(B\left(x_{0}\right.\right.$, $r))>0$, and the previous equality therefore shows that $I_{r}^{-\infty}(\mu)=\mu\left(B\left(x_{0}, r\right)\right)>$ 0 .

\section{Lemma 4.3.}

(1) For $\mu \in P_{G H P}$, we have

$$
f(\mu)=-\sum_{m \geq 1} \frac{1}{m} f_{m}(\mu)
$$

(2) For $\mu \in P_{\text {GHP }}$, we have

$$
g(\mu)=-\sum_{m \geq 1} \frac{1}{m} g_{m}(\mu) .
$$

Proof. (1) Since $\log (1-t)=-\sum_{m \geq 1} \frac{1}{m} t^{m}$ for $|t|<1$ and $\mu(\operatorname{supp} \mu \backslash B(x, r))<1$ for all $x \in \operatorname{supp} \mu$, we conclude that

$$
\log \mu(B(x, r))=\log (1-\mu(\operatorname{supp} \mu \backslash B(x, r)))=-\sum_{m \geq 1} \frac{1}{m} \mu(\operatorname{supp} \mu \backslash B(x, r))^{m}
$$

for all $x \in \operatorname{supp} \mu$. Next, because $\frac{1}{m} \mu(\operatorname{supp} \mu \backslash B(x, r))^{m} \geq 0$ for all $x$, it follows immediately from (4.5) and an application of the Lebesgue's Monotone Convergence theorem that

$$
\begin{aligned}
f(\mu) & =\int_{\operatorname{supp} \mu} \log \mu(B(x, r)) d \mu(x)=-\int_{\operatorname{supp} \mu} \sum_{m \geq 1} \frac{1}{m} \mu(\operatorname{supp} \mu \backslash B(x, r))^{m} d \mu(x) \\
& =-\sum_{m \geq 1} \frac{1}{m} \int_{\operatorname{supp} \mu} \mu(\operatorname{supp} \mu \backslash B(x, r))^{m} d \mu(x)=-\sum_{m \geq 1} \frac{1}{m} f_{m}(\mu) .
\end{aligned}
$$

This completes the proof.

(2) The proof of Part (2) is similar to the proof of Part (1), and for the sake of brevity we have therefore decided to omit the proof.

\section{Lemma 4.4 .}

(1) The function $f_{m}: P_{G H P} \rightarrow \mathbf{R}$ is upper semi-continuous.

(2) The function $g_{m}: P_{G H P} \rightarrow \mathbf{R}$ is lower semi-continuous.

Proof. (1) Let $\mu \in P_{\mathrm{GHP}}$ and let $\mu_{n} \in P_{\mathrm{GHP}}$ for $n \in \mathbf{N}$ with $\mu_{n} \rightarrow \mu$ with respect to $d_{\mathrm{GHP}}$. We must now show that $\lim _{\sup _{n}} f_{m}\left(\mu_{n}\right) \leq f_{m}(\mu)$.

We first note that we may clearly assume that there is a compact metric space $X$ such that $\mu \in \mathcal{P}(X)$ and $\mu_{n} \in \mathcal{P}(X)$ for $n \in \mathbf{N}$. Next, define $F_{m}: X^{m+1} \rightarrow \mathbf{R}$ by

$$
F_{m}\left(y_{1}, \ldots, y_{m}, x\right)=1_{\Pi_{i=1}^{m}(X \backslash B(x, r))}\left(y_{1}, \ldots, y_{m}\right)
$$

(above we have used the following notation, namely, if $A$ is a subset of a set $M$, then we will write $1_{A}: M \rightarrow \mathbf{R}$ for the indicator function on $A$ ), and note that for all 
$\nu \in P(X)$, we have

$$
\begin{aligned}
f_{m}(\nu) & =\int_{\operatorname{supp} \nu} \nu(\operatorname{supp} \nu \backslash B(x, r))^{m} d \nu(x)=\int \nu(X \backslash B(x, r))^{m} d \nu(x) \\
& =\int\left(\prod_{i=1}^{m} \int 1_{X \backslash B(x, r)}\left(y_{i}\right) d \nu\left(y_{i}\right)\right) d \nu(x) \\
& =\int \prod_{i=1}^{m} 1_{X \backslash B(x, r)}\left(y_{i}\right) d \nu^{m+1}\left(y_{1}, \ldots, y_{m}, x\right) \\
& =\int 1_{\prod_{i=1}^{m}(X \backslash B(x, r))}\left(y_{1}, \ldots, y_{m}\right) d \nu^{m+1}\left(y_{1}, \ldots, y_{m}, x\right)=\int F_{m} d \nu^{m+1}
\end{aligned}
$$

Before proving the statement in the proposition, we prove the following two claims.

Claim 1. The function $F_{m}$ is upper semi-continuous.

Proof of Claim 1. Let $\left(y_{1, n}, \ldots, y_{m, n}, x_{n}\right)_{n}$ be a sequence in $X^{m+1}$ and let $\left(y_{1}, \ldots\right.$, $\left.y_{m}, x\right) \in X^{m+1}$ such that $\left(y_{1, n}, \ldots, y_{m, n}, x_{n}\right) \rightarrow\left(y_{1}, \ldots, y_{m}, x\right)$. We must now prove that

$$
\limsup _{n} F_{m}\left(y_{1, n}, \ldots, y_{m, n}, x_{n}\right) \leq F_{m}\left(y_{1}, \ldots, y_{m}, x\right) .
$$

If $\left(y_{1}, \ldots, y_{m}\right) \in \prod_{i=1}^{m}(X \backslash B(x, r))$, then (4.7) is clearly satisfied, and we may therefore assume that $\left(y_{1}, \ldots, y_{m}\right) \notin \prod_{i=1}^{m}(X \backslash B(x, r))$. Since $\left(y_{1}, \ldots, y_{m}\right) \notin \prod_{i=1}^{m}(X \backslash$ $B(x, r))$, we conclude that there is an index $k$ such that $y_{k} \notin X \backslash B(x, r)$, whence $y_{k} \in B(x, r)$, and we can therefore choose a positive number $s>0$ such that $B\left(y_{k}, s\right) \subseteq B(x, r)$. Also, since $\left(y_{1, n}, \ldots, y_{m, n}, x_{n}\right) \rightarrow\left(y_{1}, \ldots, y_{m}, x\right)$, we can now choose a positive integer $N$ such that for all $n \geq N$, we have

$$
B\left(y_{k}, s\right) \subseteq B\left(x_{n}, r\right)
$$

and

$$
y_{k, n} \in B\left(y_{k}, s\right) .
$$

Consequently, for $n \geq N$, we have $y_{k, n} \in B\left(y_{k}, s\right) \subseteq B\left(x_{n}, r\right)$, and so $y_{k, n} \notin$ $X \backslash B\left(x_{n}, r\right)$, whence $\left(y_{1, n}, \ldots, y_{m, n}\right) \notin \prod_{i=1}^{m}\left(X \backslash B\left(x_{n}, r\right)\right)$. This clearly implies that if $n$ is a positive integer with $n \geq N$, then we have $\lim \sup _{n} F_{m}\left(y_{1, n}, \ldots, x_{m, n}, y_{n}\right)=$ $1_{\Pi_{i=1}^{m}\left(X \backslash B\left(x_{n}, r\right)\right)}\left(y_{1, n}, \ldots, y_{m, n}\right)=0$, and so $\lim \sup _{n} F_{m}\left(y_{1, n}, \ldots, x_{m, n}, y_{n}\right)=0 \leq F_{m}\left(y_{1}\right.$, $\left.\ldots, y_{m}, x\right)$. This completes the proof of Claim 1 .

Claim 2. $\mu_{n}^{m+1} \rightarrow \mu^{m+1}$ with respect to the weak topology.

Proof of Claim 2. Since $X$ is separable (because $X$ is compact) and $\mu_{n} \rightarrow \mu$ with respect to $d_{\mathrm{GHP}}$ and therefore, in particularly, with respect to the weak topology, it follows from [Bil, p. 21, Theorem 3.2] that $\mu_{n}^{m+1} \rightarrow \mu^{m+1}$ with respect to the weak topology. This completes the proof of Claim 2.

We can now prove the statement in the proposition. Indeed, it follows immediately from (4.6), Claim 1 and Claim 2 that

$$
\limsup _{n} f_{m}\left(\mu_{n}\right)=\limsup _{n} \int F_{m} d \mu_{n}^{m+1} \leq \int F_{m} d \mu^{m+1}=f_{m}(\mu)
$$

This completes the proof.

(2) The proof of Part (2) is similar to the proof of Part (1), and for the sake of brevity we have therefore decided to omit the proof. 
Lemma 4.5. Let $\mu \in P_{G H P}$ and $\mu_{n} \in P_{G H P}$ for $n \in \mathbf{N}$ with $\mu_{n} \rightarrow \mu$ with respect to $d_{G H P}$.

(1) We have

$$
\limsup _{n} \sum_{m \geq M} \frac{1}{m} f_{m}\left(\mu_{n}\right) \rightarrow 0 \text { as } M \rightarrow \infty,
$$

and

$$
\limsup _{n} \sum_{m \geq 1} \frac{1}{m} f_{m}\left(\mu_{n}\right) \leq \sum_{m \geq 1} \frac{1}{m} \limsup _{n} f_{m}\left(\mu_{n}\right) .
$$

(2) We have

$$
\liminf _{n} \sum_{m \geq 1} \frac{1}{m} g_{m}\left(\mu_{n}\right) \geq \sum_{m \geq 1} \frac{1}{m} \liminf _{n} g_{m}\left(\mu_{n}\right) .
$$

Proof. (1) We first show that $\limsup _{n} \sum_{m \geq M} \frac{1}{m} f_{m}\left(\mu_{n}\right) \rightarrow 0$ as $M \rightarrow \infty$. For brevity write $L_{M, n}=\sum_{m \geq M} \frac{1}{m} f_{m}\left(\mu_{n}\right)$. We must now prove that $\lim \sup _{n} L_{M, n} \rightarrow 0$ as $M \rightarrow \infty$. Define the function $h_{n}: \operatorname{supp} \mu_{n} \rightarrow \mathbf{R}$ by $h_{n}(x)=\mu_{n}\left(\operatorname{supp} \mu_{n} \backslash B(x, r)\right)$, and note that

$$
\begin{aligned}
L_{M, n} & =\sum_{m \geq M} \frac{1}{m} f_{m}\left(\mu_{n}\right)=\sum_{m \geq M} \frac{1}{m} \int_{\operatorname{supp} \mu_{n}} \mu_{n}\left(\operatorname{supp} \mu_{n} \backslash B(x, r)\right)^{m} d \mu_{n}(x) \\
& =\sum_{m \geq M} \frac{1}{m} \int_{\operatorname{supp} \mu_{n}} h_{n}^{m} d \mu_{n} \leq \sum_{m \geq M} \frac{1}{m}\left\|h_{n}\right\|_{\infty}^{m} \leq \frac{1}{M} \sum_{m \geq 0}\left\|h_{n}\right\|_{\infty}^{m}=\frac{1}{M} \frac{1}{1-\left\|h_{n}\right\|_{\infty}} .
\end{aligned}
$$

We conclude from this that

$$
\limsup _{n} L_{M, n} \leq \frac{1}{M} \limsup _{n} \frac{1}{1-\left\|h_{n}\right\|_{\infty}}=\frac{1}{M} \frac{1}{\liminf _{n}\left(1-\left\|h_{n}\right\|_{\infty}\right)} .
$$

However, we clearly have

$$
\begin{aligned}
1-\left\|h_{n}\right\|_{\infty} & =1-\sup _{x \in \operatorname{supp} \mu_{n}} \mu_{n}\left(\operatorname{supp} \mu_{n} \backslash B(x, r)\right) \\
& =1-\sup _{x \in \operatorname{supp} \mu_{n}}\left(1-\mu_{n}(B(x, r))\right) \\
& =\inf _{x \in \operatorname{supp} \mu_{n}}\left(1-\left(1-\mu_{n}(B(x, r))\right)\right) \\
& \left.=\inf _{x \in \operatorname{supp} \mu_{n}} \mu_{n}(B(x, r))\right)=I_{r}^{-\infty}\left(\mu_{n}\right) ;
\end{aligned}
$$

recall, that the function $I_{r}^{-\infty}: P_{\mathrm{GHP}} \rightarrow \mathbf{R}$ is defined in Section 3. In particular, combining (4.8) and (4.9), we now deduce that

$$
\limsup _{n} L_{M, n} \leq \frac{1}{M} \frac{1}{\liminf _{n} I_{r}^{-\infty}\left(\mu_{n}\right)} .
$$

Next, since $\mu_{n} \rightarrow \mu$ with respect to $d_{\mathrm{GHP}}$ and since Proposition 3.3 shows that the function $I_{r}^{-\infty}: P_{\mathrm{GHP}} \rightarrow \mathbf{R}$ is lower semi-continuous, it follows that $I_{r}^{-\infty}(\mu) \leq$ $\liminf _{n} I_{r}^{-\infty}\left(\mu_{n}\right)$, and we therefore conclude from (4.10) that

$$
\limsup _{n} L_{M, n} \leq \frac{1}{M} \frac{1}{I_{r}^{-\infty}(\mu)} .
$$

Finally, since it follows from Lemma 4.2 that $I_{r}^{-\infty}(\mu)>0$ (and so $\frac{1}{I_{r}^{-\infty}(\mu)}<\infty$ ), we now deduce from (4.11) that $\lim _{\sup _{n}} L_{M, n} \rightarrow 0$ as $M \rightarrow \infty$. 
Next, we show that $\lim \sup _{n} \sum_{m \geq 1} \frac{1}{m} f_{m}\left(\mu_{n}\right) \leq \sum_{m>1} \frac{1}{m} \lim \sup _{n} f_{m}\left(\mu_{n}\right)$. However, this statement follows immediately from Lemma 4.1 and the fact that lim $\sup _{n}$ $\sum_{m>M} \frac{1}{m} f_{m}\left(\mu_{n}\right) \rightarrow 0$ as $M \rightarrow \infty$.

(2) This statement follows immediately from Lemma 4.1.

Lemma 4.6.

(1) The function $f: P_{G H P} \rightarrow \mathbf{R}$ is lower semi-continuous.

(2) The function $g: P_{G H P} \rightarrow \mathbf{R}$ is upper semi-continuous.

Proof. (1) Let $\mu \in P_{\mathrm{GHP}}$ and $\mu_{n} \in P_{\mathrm{GHP}}$ for $n \in \mathbf{N}$ with $\mu_{n} \rightarrow \mu$ with respect to $d_{\mathrm{GHP}}$. We must now prove that $f(\mu) \leq \liminf _{n} f\left(\mu_{n}\right)$. However, combining Lemma 4.3.(1), Lemma 4.4.(1) and Lemma 4.5.(1) we immediately conclude that

$$
\begin{aligned}
\liminf _{n} f\left(\mu_{n}\right) & =\liminf _{n}-\sum_{m \geq 1} \frac{1}{m} f_{m}\left(\mu_{n}\right) & & \text { [by Lemma 4.3.(1)] } \\
& =-\limsup _{n} \sum_{m \geq 1} \frac{1}{m} f_{m}\left(\mu_{n}\right) & & \\
& \geq-\sum_{m \geq 1} \frac{1}{m} \limsup _{n} f_{m}\left(\mu_{n}\right) & & \text { [by Lemma 4.5.(1)] } \\
& \geq-\sum_{m \geq 1} \frac{1}{m} f_{m}(\mu) & & \text { [by Lemma 4.4.(1)] } \\
& =f(\mu) . & & \text { [by Lemma 4.3.(1)] }
\end{aligned}
$$

This completes the proof of Part (1).

(2) Let $\mu \in P_{\mathrm{GHP}}$ and $\mu_{n} \in P_{\mathrm{GHP}}$ for $n \in \mathbf{N}$ with $\mu_{n} \rightarrow \mu$ with respect to $d_{\mathrm{GHP}}$. We must now prove that $g(\mu) \geq \limsup _{n} g\left(\mu_{n}\right)$. Similar to the proof of Part (1), combining Lemma 4.3.(2), Lemma 4.4.(2) and Lemma 4.5.(2) we now conclude that

$$
\begin{aligned}
\limsup _{n} g\left(\mu_{n}\right) & =\limsup _{n}-\sum_{m \geq 1} \frac{1}{m} g_{m}\left(\mu_{n}\right) \\
& =-\liminf _{n} \sum_{m \geq 1} \frac{1}{m} g_{m}\left(\mu_{n}\right) \\
& \leq-\sum_{m \geq 1} \frac{1}{m} \liminf _{n} g_{m}\left(\mu_{n}\right) \\
& \leq-\sum_{m \geq 1} \frac{1}{m} g_{m}(\mu) \\
& =g(\mu) .
\end{aligned}
$$

[by Lemma 4.3.(2)]

This completes the proof of Part (2).

We can now prove the main semi-continuity result in the section.

\section{Proposition 4.7.}

(1) The function $I_{r}^{1}: P_{\mathrm{GHP}} \rightarrow \mathbf{R}$ is lower semi-continuous on $P_{\mathrm{GHP}}$ for all $r>0$.

(2) The function $J_{r}^{1}: P_{\mathrm{GHP}} \rightarrow \mathbf{R}$ is upper semi-continuous on $P_{\mathrm{GHP}}$ for all $r>0$.

Proof. This follows from Lemma 4.6 since $I_{r}^{1}(\mu)=\exp f(\mu)$ and $J_{r}^{1}(\mu)=\exp g(\mu)$ for all $\mu \in P_{\mathrm{GHP}}$.

Finally, we can prove Theorem 1.5. 
Proof of Theorem 1.5. This result follows immediately from Proposition 4.7.

We finish this section by proving the following useful approximation result.

Lemma 4.8. Let $X$ be a compact metric space and $\mu \in \mathcal{P}(X)$. Let $r>0$. Then there is a measure $P \in \mathcal{P}(X)$ with finite support satisfying supp $P \subseteq \operatorname{supp} \mu$ such that $d_{H}(\operatorname{supp} \mu, \operatorname{supp} P)<r$ and $d_{P}(\mu, P)<r$ (recall that the Hausdorff metric $d_{H}$ is defined in (1.2) and that the Prohoroff metric $d_{P}$ is defined in (1.3)).

Proof. The proof of this lemma is given in [Ols3, Lemma 3.3].

\section{Proof of Theorem 1.3 Equation (1.23): $\underline{D}_{\Pi}^{1}(\mu)=0$ for a typical $\mu \in P_{\mathrm{GHP}}$}

In this section we prove Theorem 1.3 Equation (1.23). The first lemma (i.e. Lemma 5.1) is standard; however, for the benefit of the reader we have decided to state it explicitly.

Lemma 5.1. The Reverse Fatou's Lemma. [Str, Theorem 3.2.3] Let $(M, \mathcal{E}, \mu)$ be a measure space and let $\left(\varphi_{n}\right)_{n}$ be a sequence of positive measurable functions $\varphi_{n}: M \rightarrow[0, \infty]$. If $\int \sup _{n} \varphi_{n} d \mu<\infty$, then $\lim \sup _{n} \int \varphi_{n} d \mu \leq \int \lim \sup _{n} \varphi_{n} d \mu$.

Lemma 5.2. Let $\Pi=\left(\Pi_{t}\right)_{t \geq t_{0}}$ be an averaging system. Let $c \in \mathbf{R}$ and $t \geq t_{0}$. Then the set

$$
\left\{\mu \in P_{G H P} \mid \int \frac{\log I_{e^{-s}}^{1}(\mu)}{-s} d \Pi_{t}(s)<c\right\}
$$

is open in $P_{G H P}$.

Proof. Write

$$
\begin{aligned}
F & =P_{\mathrm{GHP}} \backslash\left\{\mu \in P_{\mathrm{GHP}} \mid \int \frac{\log I_{e^{-s}}^{1}(\mu)}{-s} d \Pi_{t}(s)<c\right\} \\
& =\left\{\mu \in P_{\mathrm{GHP}} \mid \int \frac{\log I_{e^{-s}}^{1}(\mu)}{-s} d \Pi_{t}(s) \geq c\right\} .
\end{aligned}
$$

We must now prove that $F$ is closed in $P_{\mathrm{GHP}}$. In order to show this, we fix a sequence $\left(\mu_{n}\right)_{n}$ in $F$ and $\mu \in P_{\mathrm{GHP}}$ with $\mu_{n} \rightarrow \mu$. We must now prove that $\mu \in F$, i.e. we must prove that $\int \frac{\log I_{e^{-s}}^{1}(\mu)}{-s} d \Pi_{t}(s) \geq c$. For brevity define functions $\varphi, \varphi_{n}:\left[t_{0}, \infty\right) \rightarrow$ $[0, \infty)$ by $\varphi(s)=\frac{\log I_{e^{-s}}^{1}(\mu)}{-s}$ and $\varphi_{n}(s)=\frac{\log I_{e^{-s}}^{1}\left(\mu_{n}\right)}{-s}$. We now prove the following three claims.

Claim 1. For all $s \geq t_{0}$, we have $\varphi(s) \geq \lim \sup _{n} \varphi_{n}(s)$. In particular $\int \varphi d \Pi_{t} \geq$ $\int \lim \sup _{n} \varphi_{n} d \Pi_{t}$.

Proof of Claim 1. It follows from Proposition 4.7 that the function $I_{r}^{1}: P_{\mathrm{GHP}} \rightarrow \mathbf{R}$ is lower semi-continuous for all $r>0$, and we therefore conclude that $I_{e^{-s}}^{1}(\mu) \leq$ $\lim \inf _{n} I_{e^{-s}}^{1}\left(\mu_{n}\right)$. We immediately deduce from this that

$$
\varphi(s)=\frac{\log I_{e^{-s}}^{1}(\mu)}{-s} \geq \frac{\log \liminf I_{n} I_{e^{-s}}^{1}\left(\mu_{n}\right)}{-s}=\limsup _{n} \frac{\log I_{e^{-s}}^{1}\left(\mu_{n}\right)}{-s}=\limsup _{n} \varphi_{n}(s) .
$$

This completes the proof of Claim 1.

Claim 2. We have $\int \sup _{n} \varphi_{n} d \Pi_{t}<\infty$.

Proof of Claim 2. The measure $\Pi_{t}$ has compact support and we can therefore choose $T_{0} \geq t_{0}$ such that $\operatorname{supp} \Pi_{t} \in\left[t_{0}, T_{0}\right]$. Next, since clearly $I_{e^{-T_{0}}}^{1}(\mu)>$ 
0 and $I_{e^{-T_{0}}}^{1}: P_{\mathrm{GHP}} \rightarrow \mathbf{R}$ is lower semi-continuous (by Proposition 4.7), we conclude that $0<I_{e^{-T_{0}}}^{1}(\mu) \leq \liminf _{n} I_{e^{-T_{0}}}^{1}\left(\mu_{n}\right)$, and we therefore deduce that $c_{0}=$ $\inf _{n} I_{e^{-T_{0}}}^{1}\left(\mu_{n}\right)>0$. It follows from this that for all $s \in\left[t_{0}, T_{0}\right]$, we have $\sup _{n} \varphi_{n}(s)=$ $\sup _{n} \frac{\log I_{e^{-s}}^{1}\left(\mu_{n}\right)}{-s}=\sup _{n} \frac{\log I_{e^{-s}}^{1}\left(\mu_{n}\right)^{-1}}{s} \leq \sup _{n} \frac{\log I_{e^{-} T_{0}}^{1}\left(\mu_{n}\right)^{-1}}{t_{0}} \leq \frac{\log c_{0}^{-1}}{t_{0}}$. This clearly implies that $\int \sup _{n} \varphi_{n} d \Pi_{t}=\int_{t_{0}}^{T_{0}} \sup _{n} \varphi_{n} d \Pi_{t} \leq \int_{t_{0}}^{T_{0}} \frac{\log c_{0}^{-1}}{t_{0}} d \Pi_{t}=\Pi_{t}\left(\left[t_{0}, T_{0}\right]\right) \frac{\log c_{0}^{-1}}{t_{0}}<\infty$. This completes the proof of Claim 2.

Claim 3. We have $\int \lim \sup _{n} \varphi_{n} d \Pi_{t} \geq c$.

Proof of Claim 3. Since $\mu_{n} \in F$, we conclude that $\int \varphi_{n} d \Pi_{t}=\int \frac{\log I_{e^{-s}}^{1}\left(\mu_{n}\right)}{-s} d \Pi_{t}(s) \geq$ $c$ for all $n$, whence

$$
c \leq \limsup _{n} \int \varphi_{n} d \Pi_{t}
$$

We also note that since $\varphi_{n} \geq 0$, it follows from Claim 2 and the Reverse Fatou's lemma (Lemma 4.1) that

$$
\limsup _{n} \int \varphi_{n} d \Pi_{t} \leq \int \underset{n}{\lim \sup } \varphi_{n} d \Pi_{t} .
$$

Combining the (5.1) and (5.2) we now conclude that

$$
c \leq \limsup _{n} \int \varphi_{n} d \Pi_{t} \leq \int \limsup _{n} \varphi_{n} d \Pi_{t} .
$$

This completes the proof of Claim 3.

Finally, we deduce from Claim 1 and Claim 3 that

$$
\int \frac{\log I_{e^{-s}}^{1}(\mu)}{-s} d \Pi_{t}(s)=\int \varphi d \Pi_{t} \geq \int \limsup _{n} \varphi_{n} d \Pi_{t} \geq c .
$$

This completes the proof.

We now turn towards the proof of Theorem 1.3 Equation (1.23).

Proof of Theorem 1.3 Equation (1.23). We must prove that for a typical measure $\mu \in P_{\mathrm{GHP}}$, we have $\underline{D}_{\Pi}^{1}(\mu)=0$. Since $\underline{D}_{\Pi}^{1}(\mu) \geq 0$, it suffices to prove that the set

$$
U=\left\{\mu \in P_{\mathrm{GHP}} \mid \underline{D}_{\Pi}^{1}(\mu)>0\right\}
$$

is meagre. For $u>0$, write

$$
U_{u}=\left\{\mu \in P_{\mathrm{GHP}} \mid \underline{D}_{\Pi}^{1}(\mu)>u\right\} .
$$

Since

$$
U=\bigcup_{\substack{u \in \mathbf{Q} \\ u>0}} U_{u},
$$

it suffices to show that $U_{u}$ is meagre for all $u \in \mathbf{Q}$ with $u>0$.

We therefore fix $u \in \mathbf{Q}$ with $u>0$. Next, in order to show that $U_{u}$ is meagre, we note that it suffices to show that there is a countable family $\left(G_{k}\right)_{k}$ of open and dense subsets of $P_{\mathrm{GHP}}$ with $\cap_{k} G_{k} \subseteq P_{\mathrm{GHP}} \backslash U_{u}$. We now construct the sets $G_{k}$. For $t \geq t_{0}$, let

$$
L_{t}=\left\{\mu \in P_{\mathrm{GHP}} \mid \int \frac{\log I_{e^{-s}}^{1}(\mu)}{-s} d \Pi_{t}(s)<u\right\}
$$


and for each positive integer $k$, put

$$
G_{k}=\bigcup_{t \geq k} L_{t}
$$

Below we show that the family $\left(G_{k}\right)_{k}$ consists of open and dense subsets of $P_{\mathrm{GHP}}$ with $\cap_{k} G_{k} \subseteq P_{\mathrm{GHP}} \backslash U_{u}$; this is the content of the following three claims.

Claim 1. The set $G_{k}$ is open in $P_{G H P}$.

Proof of Claim 1. Indeed, since it follows from Lemma 5.2 that $L_{t}$ is open for all $t \geq t_{0}$, we immediately conclude that $G_{k}=\bigcup_{t \geq k} L_{t}$ is open. This completes the proof of Claim 1.

Claim 2. The set $G_{k}$ is dense in $P_{G H P}$.

Proof of Claim 2. Let $\mu \in P_{\mathrm{GHP}}$ and let $r>0$. We must now find $\nu \in P_{\mathrm{GHP}}$ such that $d_{\mathrm{GHP}}(\mu, \nu)<r$ and $\nu \in G_{k}$. Writing $X=\operatorname{supp} \mu$, it follows from Lemma 4.8 that we can find a measure $\nu \in \mathcal{P}(X)$ with finite support such that $d_{\mathrm{H}}(\operatorname{supp} \mu, \operatorname{supp} \nu)<\frac{r}{2}$ and $d_{\mathrm{P}}(\mu, \nu)<\frac{r}{2}$.

In particular, we conclude that $d_{\mathrm{GHP}}(\mu, \nu) \leq d_{\mathrm{H}}(\operatorname{supp} \mu, \operatorname{supp} \nu)+d_{\mathrm{P}}(\mu, \nu)<$ $\frac{r}{2}+\frac{r}{2}=r$.

Next, we show that $\nu \in G_{k}$. Indeed, since the support of $\nu$ is finite, there is a finite subset $E$ of $X$ and a probability vector $\left(p_{x}\right)_{x \in E}$ such that $\nu=\sum_{x \in E} p_{x} \delta_{x}$, whence

$$
I_{e^{-t}}^{1}(\nu)=\exp \int \log \sum_{y \in E} p_{y} \delta_{y}\left(B\left(x, e^{-t}\right)\right) d \nu(x)
$$

for all $t>0$. Next, write $r_{E}=\min _{x_{1}, x_{2} \in E, x_{1} \neq x_{2}}\left|x_{1}-x_{2}\right|$, and note that $r_{E}>0$ because $E$ is finite. Choose $t_{E}$ such that $e^{-t_{E}}=r_{E}$ and note that it follows from the definition of $r_{E}$ that $\log \sum_{y \in E} p_{y} \delta_{y}\left(B\left(x, e^{-t}\right)\right)=\sum_{y \in E}\left(\log p_{y}\right) \delta_{y}\left(B\left(x, e^{-t}\right)\right)$ for all $x \in X$ and all $t \geq t_{E}$. We conclude from this and (5.3) that

$$
\begin{aligned}
I_{e^{-t}}^{1}(\nu) & =\exp \sum_{y \in E}\left(\log p_{y}\right) \int \delta_{y}\left(B\left(x, e^{-t}\right)\right) d \nu(x) \\
& =\exp \sum_{y \in E}\left(\log p_{y}\right) \sum_{x \in E} p_{x} \delta_{y}\left(B\left(x, e^{-t}\right)\right)
\end{aligned}
$$

for all $t \geq t_{E}$. However, since $\sum_{x \in E} p_{x} \delta_{y}\left(B\left(x, e^{-t}\right)\right)=p_{y}$ for all $y \in E$ and all $t \geq t_{E}$, we deduce from (5.4) that

$$
I_{e^{-t}}^{1}(\nu)=\exp \sum_{y \in E} p_{y} \log p_{y}
$$

for all $t \geq t_{E}$. It follows from (5.5) that $\frac{\log I_{e-t}^{q}(\nu)}{-t} \rightarrow 0$, and the consistency condition therefore implies that $\int \frac{\log I_{e^{-s}}^{1}(\nu)}{-s} d \Pi_{t}(s) \rightarrow 0$ as $t \rightarrow \infty$. We conclude immediately from this and the fact that $u>0$ that there is a real number $t \geq k$ such that $\int \frac{\log I_{e-s}^{1}(\nu)}{-s} d \Pi_{t}(s)<u$, and so $\nu \in L_{t} \subseteq G_{k}$. This completes the proof of Claim 2 .

Claim 3. We have $\cap_{k} G_{k} \subseteq P_{G H P} \backslash U_{u}$.

Proof of Claim 3. Let $\mu \in \cap_{k} G_{k}$. Hence for each positive integer $k$, we can find $t_{k} \geq k$ such that $\mu \in L_{t_{k}}$, whence $\int \frac{\log I_{e^{-s}}^{1}(\mu)}{-s} d \Pi_{t_{k}}(s)<u$ for all positive integers $k$. We conclude from this that $\underline{D}_{\Pi}^{1}(\mu)=\liminf _{t \rightarrow \infty} \int \frac{\log I_{e^{-s}}^{1}(\mu)}{-s} d \Pi_{t}(s) \leq$ 
$\liminf _{k} \int \frac{\log I_{e^{-s}}^{1}(\mu)}{-s} d \Pi_{t_{k}}(s) \leq u$, whence $\mu \in P_{\mathrm{GHP}} \backslash U_{u}$. This completes the proof of Claim 3.

Combining Claim 1, Claim 2 and Claim 3, we now conclude that $U_{u}$ is meagre.

\section{Proof of Theorem 1.4 Equation (1.26): $\bar{D}_{\Pi}^{\infty}(\boldsymbol{\mu})=\infty$ for a typical $\boldsymbol{\mu} \in \boldsymbol{P}_{\mathrm{GHP}}$.}

In this section we prove Theorem 1.4 equation (1.26).

Lemma 6.1. Let $\Pi=\left(\Pi_{t}\right)_{t \geq t_{0}}$ be an averaging system. Let $c \in \mathbf{R}$ and $t \geq t_{0}$. Then the set

$$
\left\{\mu \in P_{G H P} \mid \int \frac{\log J_{e^{-s}}^{\infty}(\mu)}{-s} d \Pi_{t}(s)>c\right\}
$$

is open in $P_{G H P}$.

Proof. Write

$$
\begin{aligned}
F & =P_{\mathrm{GHP}} \backslash\left\{\mu \in P_{\mathrm{GHP}} \mid \int \frac{\log J_{e^{-s}}^{\infty}(\mu)}{-s} d \Pi_{t}(s)>c\right\} \\
& =\left\{\mu \in P_{\mathrm{GHP}} \mid \int \frac{\log J_{e^{-s}}^{\infty}(\mu)}{-s} d \Pi_{t}(s) \leq c\right\} .
\end{aligned}
$$

We must now prove that $F$ is closed in $P_{\mathrm{GHP}}$. In order to show this, we fix a sequence $\left(\mu_{n}\right)_{n}$ in $F$ and $\mu \in P_{\mathrm{GHP}}$ with $\mu_{n} \rightarrow \mu$. We must now prove that $\mu \in F$, i.e. we must prove that $\int \frac{\log J_{e^{-s}}^{\infty}(\mu)}{-s} d \Pi_{t}(s) \leq c$. For brevity define functions $\varphi, \varphi_{n}:\left[t_{0}, \infty\right) \rightarrow$ $[0, \infty)$ by $\varphi(s)=\frac{\log J_{e^{-s}}^{\infty}(\mu)}{-s}$ and $\varphi_{n}(s)=\frac{\log J_{e^{-s}}^{\infty}\left(\mu_{n}\right)}{-s}$. We now prove the following two claims.

Claim 1. We have $\int \liminf _{n} \varphi_{n} d \Pi_{t} \leq c$.

Proof of Claim 1. Since $\mu_{n} \in F$, we conclude that $\int \varphi_{n} d \Pi_{t}=\int \frac{\log J_{e^{-s}}^{q}\left(\mu_{n}\right)}{s} d \Pi_{t}(s)$ $\leq c$ for all $n$, whence $\liminf _{n} \int \varphi_{n} d \Pi_{t} \leq c$. Also, since $\varphi_{n} \geq 0$, it therefore follows from Fatou's lemma that $\int \liminf _{n} \varphi_{n} d \Pi_{t} \leq \liminf _{n} \int \varphi_{n} d \Pi_{t} \leq c$. This completes the proof of Claim 2.

Claim 2. For all $s \geq t_{0}$, we have $\varphi(s) \leq \liminf _{n} \varphi_{n}(s)$. In particular $\int \varphi d \Pi_{t} \leq$ $\int \liminf \varphi_{n} d \Pi_{t}$.

Proof of Claim 2. It follows from Proposition 3.2 that the function $J_{r}^{\infty}: P_{\mathrm{GHP}} \rightarrow$ $\mathbf{R}$ is upper semi-continuous for all $r>0$, whence $\lim \sup _{n} J_{e^{-s}}^{\infty}\left(\mu_{n}\right) \leq J_{e^{-s}}^{\infty}(\mu)$. We conclude immediately from this that

$$
\varphi(s)=\frac{\log J_{e^{-s}}^{\infty}(\mu)}{-s} \leq \frac{\log \lim \sup _{n} J_{e^{-s}}^{\infty}\left(\mu_{n}\right)}{-s}=\liminf _{n} \frac{\log J_{e^{-s}}^{\infty}\left(\mu_{n}\right)}{-s}=\liminf _{n} \varphi_{n}(s) .
$$

This completes the proof of Claim 2 .

Finally, we deduce from Claim 1 and Claim 2 that

$$
\int \frac{\log J_{e^{-s}}^{\infty}(\mu)}{s} d \Pi_{t}(s)=\int \varphi d \Pi_{t} \leq \int \liminf _{n} \varphi_{n} d \Pi_{t} \leq c .
$$

This completes the proof.

We now turn towards the proofs of Theorem 1.4 Equation (1.26). 
Proof of Theorem 1.4 Equation (1.26). We must prove that for a typical measure $\mu \in P_{\mathrm{GHP}}$, we have $\bar{D}_{\Pi}^{\infty}(\mu)=\infty$, i.e. we must prove that the set

$$
U=\left\{\mu \in \mathcal{P}(X) \mid \bar{D}_{\Pi}^{\infty}(\mu)<\infty\right\}
$$

is meagre. For $u \in \mathbf{R}$, write

$$
U_{u}=\left\{\mu \in \mathcal{P}(X) \mid \bar{D}_{\Pi}^{\infty}(\mu)<u\right\} .
$$

Since

$$
U=\bigcup_{u \in \mathbf{Q}} U_{u}
$$

it suffices to show that $U_{u}$ is meagre for all $u \in \mathbf{Q}$ with $u>0$.

We therefore fix $u \in \mathbf{Q}$ with $u>0$, and note that it suffices to show that there is a countable family $\left(G_{k}\right)_{k}$ of open and dense subsets of $P_{\mathrm{GHP}}$ with $\cap_{k} G_{k} \subseteq P_{\mathrm{GHP}} \backslash U_{u}$. We now construct the sets $G_{k}$. For $t \geq t_{0}$, let

$$
L_{t}=\left\{\mu \in P_{\mathrm{GHP}} \mid \int \frac{\log J_{e^{-s}}^{\infty}(\mu)}{-s} d \Pi_{t}(s)>u\right\},
$$

and for each positive integer $k$, put

$$
G_{k}=\bigcup_{t \geq k} L_{t}
$$

Below we show that the family $\left(G_{k}\right)_{k}$ consists of open and dense subsets of $P_{\mathrm{GHP}}$ with $\bigcap_{k} G_{k} \subseteq P_{\mathrm{GHP}} \backslash U_{u}$; this is the content of the following three claims.

Claim 1. The set $G_{k}$ is open in $P_{G H P}$.

Proof of Claim 1. Indeed, since it follows from Lemma 6.1 that $L_{t}$ is open for all $t \geq t_{0}$, we immediately conclude that $G_{k}=\cup_{t \geq k} L_{t}$ is open. This completes the proof of Claim 1.

Claim 2. The set $G_{k}$ is dense in $P_{G H P}$.

Proof of Claim 2. Let $\mu \in P_{\mathrm{GHP}}$ and $r>0$. We must now find $\nu \in P_{\mathrm{GHP}}$ with $d_{\mathrm{GHP}}(\mu, \nu)<r$ and $\nu \in G_{k}$. Write $M=\operatorname{supp} \mu$. It follows from Lemma 4.8 that there is a measure $P \in \mathcal{P}(M)$ with finite support such that $d_{\mathrm{H}}(\operatorname{supp} \mu$, supp $P)<\frac{r}{4}$ and $d_{\mathrm{P}}(\mu, P)<\frac{r}{4}$. Also, we can find a positive integer $N$ with $N>u$. We now write $C=\left\{x \in \mathbf{R}^{N}|| x \mid \leq \frac{r}{4}\right\}$ and let $\lambda$ denote the normalized $N$-dimensional Lebesgue measure restricted to $C$. Finally, put

$$
\nu=P \times \lambda .
$$

Below we prove that $d_{\mathrm{GHP}}(\mu, \nu)<r$ and $\nu \in G_{k}$.

We first prove that $d_{\mathrm{GHP}}(\mu, \nu)<r$. Let $X=\operatorname{supp} \mu \times C$ and equip $X$ with the maximum metric. Next, define $f: \operatorname{supp} \mu \rightarrow X$ and $g: \operatorname{supp} \nu=\operatorname{supp} P \times C \rightarrow X$ by $f(x)=(x, 0)$ and $g(x, y)=(x, y)$, and note that $f$ and $g$ are isometries. Next, we observe that

$$
\begin{aligned}
d_{\mathrm{P}}\left(\mu \circ f^{-1}, \nu \circ g^{-1}\right) & =\sup _{F \in \operatorname{Lip}(X)}\left|\int F d\left(\mu \circ f^{-1}\right)-\int F d\left(\nu \circ g^{-1}\right)\right| \\
& =\sup _{F \in \operatorname{Lip}(X)}\left|\int F(x, 0) d \mu(x)-\int F(x, y) d \nu(x, y)\right|
\end{aligned}
$$




$$
\begin{aligned}
& =\sup _{F \in \operatorname{Lip}(X)}\left|\int_{\operatorname{supp} \mu} F(x, 0) d \mu(x)-\int_{C} \int_{\operatorname{supp} \mu} F(x, y) d P(x) d \lambda(y)\right| \\
& =\sup _{F \in \operatorname{Lip}(X)}\left|\int_{C} \int_{\operatorname{supp} \mu} F(x, 0) d \mu(x) d \lambda(y)-\int_{C} \int_{\operatorname{supp} \mu} F(x, y) d P(x) d \lambda(y)\right| \\
& \leq \sup _{F \in \operatorname{Lip}(X)} \int_{C}\left|\int_{\operatorname{supp} \mu} F(x, 0) d \mu(x)-\int_{\operatorname{supp} \mu} F(x, y) d P(x)\right| d \lambda(y) .
\end{aligned}
$$

However, for all $F \in \operatorname{Lip}(X)$ and $y \in C$, we have

$$
\begin{aligned}
& \left|\int_{\operatorname{supp} \mu} F(x, 0) d \mu(x)-\int_{\operatorname{supp} \mu} F(x, y) d P(x)\right| \\
& \leq\left|\int_{\operatorname{supp} \mu} F(x, 0) d \mu(x)-\int_{\operatorname{supp} \mu} F(x, 0) d P(x)\right| \\
& \quad+\left|\int_{\operatorname{supp} \mu} F(x, 0) d P(x)-\int_{\operatorname{supp} \mu} F(x, y) d P(x)\right| \\
& \leq d_{\mathrm{P}}(\mu, P)+\int_{\operatorname{supp} \mu}|F(x, 0)-F(x, y)| d P(x) \\
& \leq d_{\mathrm{P}}(\mu, P)+\int_{\operatorname{supp} \mu} \operatorname{Lip}(X)|(x, 0)-(x, y)| d P(x) \\
& \leq d_{\mathrm{P}}(\mu, P)+\int_{\operatorname{supp} \mu}|y| d P(x) \leq d_{\mathrm{P}}(\mu, P)+\frac{r}{4}<\frac{r}{4}+\frac{r}{4}=\frac{r}{2} .
\end{aligned}
$$

Combining (6.1) and (6.2), we conclude that

$$
d_{\mathrm{P}}\left(\mu \circ f^{-1}, \nu \circ g^{-1}\right)<\sup _{F \in \operatorname{Lip}(X)} \int_{C} \frac{r}{2} d \lambda(y)=\frac{r}{2} .
$$

We also note that

$$
\begin{aligned}
& d_{\mathrm{H}}(f(\operatorname{supp} \mu), g(\operatorname{supp} \nu))=d_{\mathrm{H}}(\operatorname{supp} \mu \times\{0\}, \operatorname{supp} P \times C) \\
& \leq d_{\mathrm{H}}(\operatorname{supp} \mu \times\{0\}, \operatorname{supp} P \times\{0\})+d_{\mathrm{H}}(\operatorname{supp} P \times\{0\}, \operatorname{supp} P \times C) \\
& \leq d_{\mathrm{H}}(\operatorname{supp} \mu, \operatorname{supp} P)+d_{\mathrm{H}}(\{0\}, C)<\frac{r}{4}+\frac{r}{4}=\frac{r}{2} .
\end{aligned}
$$

Finally, combining (6.3) and (6.4) immediately gives

$$
d_{\mathrm{GHP}}(\mu, \nu) \leq d_{\mathrm{H}}(f(\operatorname{supp} \mu), g(\operatorname{supp} \nu))+d_{\mathrm{P}}\left(\mu \circ f^{-1}, \nu \circ g^{-1}\right)<r .
$$

Next, we prove that $\nu \in G_{k}$. Indeed, since $\lambda$ is the normalized $N$-dimensional Lebesgue measure restricted to $C=\left\{x \in \mathbf{R}^{N}|| x \mid \leq \frac{r}{4}\right\}$, a simple and straightforward calculation shows that $\frac{\log J_{r}^{\infty}(\lambda)}{\log r} \rightarrow N$ as $r \searrow 0$. It follows from this and the fact that supp $P$ is finite that $\frac{\log J_{r}^{\infty}(\nu)}{\log r}=\frac{\log J_{r}^{\infty}(P \times \lambda)}{\log r} \rightarrow N$ as $r \searrow 0$, and so $\frac{\log J_{e^{-t}}^{\infty}(\nu)}{-t} \rightarrow N$ as $t \rightarrow \infty$. We deduce from this and the consistency condition that $\int \frac{\log J_{e^{-s}}^{\infty}(\nu)}{-s} d \Pi_{t}(s) \rightarrow$ $N$ as $t \rightarrow \infty$, and since $N>u$, we can therefore find $t \geq k$ with $\int \frac{\log J_{e^{-s}}^{\infty}(\nu)}{-s} d \Pi_{t}(s)>$ $u$, whence $\nu \in L_{t} \subseteq G_{k}$. This completes the proof of Claim 2 .

Claim 3. We have $\bigcap_{k} G_{k} \subseteq P_{G H P} \backslash U_{u}$. 
Proof of Claim 3. Let $\mu \in \bigcap_{k} G_{k}$. Hence for each positive integer $k$, we can find $t_{k} \geq k$ such that $\mu \in L_{t_{k}}$, whence $\int \frac{\log J_{e^{-s}}^{\infty}(\mu)}{-s} d \Pi_{t_{k}}(s)>u$ for all positive integers $k$. Since clearly $I_{r}^{\infty}(\mu) \leq J_{r}^{\infty}(\mu)$ for all $r>0$, we immediately conclude from this that $\bar{D}_{\Pi}^{\infty}(\mu)=\lim \sup _{t \rightarrow \infty} \int \frac{\log I_{e^{-s}}^{\infty}(\mu)}{-s} d \Pi_{t}(s) \geq \lim \sup _{t \rightarrow \infty} \int \frac{\log J_{e^{-s}}^{\infty}(\mu)}{-s} d \Pi_{t}(s) \geq$ $\lim \sup _{k} \int \frac{\log J_{e^{-}}^{\infty}(\mu)}{-s} d \Pi_{t_{k}}(s) \geq u$, and so $\mu \in P_{\mathrm{GHP}} \backslash U_{u}$. This completes the proof of Claim 3.

Combining Claim 1, Claim 2 and Claim 3, we now conclude that $U_{u}$ is meagre.

Acknowledgements. The author thanks an anonymous referee for carefully reading the manuscript and for pointing out that the statement in Lemma 4.1.(2) follows immediately from Fatou's Lemma.

\section{References}

[ADH] Abraham, R., J.-F. Delmas, and P. Hoscheit: A note on the Gromov-HausdorffProkhorov distance between (locally) compact metric measure spaces. - Electron. J. Probab. 18, 2013.

[Bay1] BAyART, F.: The multifractal box dimensions of typical measures. - Fund. Math. 219, $2012,145-162$.

[Bay2] BAYART, F.: How do the typical $L^{q}$-dimensions of measures behave? - Indiana Univ. Math. J. 63, 2014, 687-726.

[Bil] Billingsley, P.: Convergence of probability measures. - Wiley, 1968.

[Bog] Bogachev, V. I.: Measure theory. Volume II. - Springer Verlag, 2007.

[Fal] Falconer, K. J.: Fractal geometry. - John Wiley \& Sons, Ltd., Chichester, 1989.

[Gen] Genyuk, J.: - A typical measure typically has no local dimension. - Real Anal. Exchange $23,1997 / 98,525-537$.

[Har] Hardy, G. H.: Divergent series. - Oxford, 1949.

[Has] HaAse, H.: A survey on the dimension of measures. - In: Topology, measures, and fractals (Warnemünde, 1991), Math. Res. 66, Akademie-Verlag, Berlin, 1992, 66-75.

[HJKPS] Halsey, T. C., M. H. Jensen, L. P. Kadanoff, I. Procaccia, and B. J. Shraiman: Fractal measures and their singularities: The characterization of strange sets - Phys. Rev. A 33, 1986, 1141-1151.

[Jac] JАCOB, M.: Über die Äquivalenz der Cesàroschen und der Hölderschen Mittel für Integrale bei gleicher reeller Ordnung $k>0$. - Math. Z. 26, 1927, 672-682.

[Mie] Miermont, G.: Tessellations of random maps of arbitrary genus. - Ann. Sci. Éc. Norm. Supér. (4) 42, 2009, 725-781.

[MR] Myjak, J., and R. Rudnicki: Typical properties of correlation dimension. - Real Anal. Exchange 28, 2002/03, 269-278.

[Ols1] Olsen, L.: Typical $L^{q}$-dimensions of measures. - Monaths. Math. 146, 2005, 143-157.

[Ols2] Olsen, L.: Typical upper $L^{q}$-dimensions of measures for $q \in[0,1]$. - Bull. Sci. Math. 132, 2008, 551-561.

[Ols3] Olsen, L.: On the average $L^{q}$-dimensions of typical measures belonging to the GromovHausdorff-Prohoroff space. - J. Math. Anal. Appl. 469, 2019, 916-934.

[Oxt] Oxтовy, J.: Measure and category. A survey of the analogies between topological and measure spaces. - Grad. Texts in Math. 2, Springer-Verlag, New York-Berlin, 1971.

[Pes] Pesin, Y.: Dimension theory in dynamical systems. Contemporary views and applications. - The University of Chicago Press, 1997. 
[Rén1] RÉNYi, A.: Some fundamental questions of information theory. - Magyar Tud. Akad. Mat. Fiz. Oszt. Közl 10, 1960, 251-282.

[Rén2] RÉNYI, A.: On measures of entropy and information. - Proceedings 4th Berkeley Symposium on Mathematical Statistics and Probability 1960, Univ. of California Press, Berkeley, $1961,547-561$.

[Str] Stroock, D.: Essentials of integration theory for analysis. - Springer Verlag, 2011.

[Vil] Villani, C.: Optimal transport: old and new - Grundlehren Math. Wiss. 338, - Springer Verlag, 2009.

[Wal] Walters, P.: An introduction to ergodic theory. - Grad. Texts in Math. 79, Springer Verlag, 2013.

Received 18 February 2019 • Accepted 9 August 2019 